\title{
A categorical presentation of quantum computation with anyons
}

\author{
Prakash Panangaden $^{1}$ and Éric Oliver Paquette ${ }^{2}$ \\ 1 School of computer science, McGill University prakash@cs.mcgill.ca \\ 2 DIRO, Université de Montréal eopaquette@inexistant.net
}

Summary. In nature one observes that in three space dimensions particles are either symmetric under interchange (bosons) or antisymmetric (fermions). These phases give rise to the two possible "statistics" that one observes. In two dimensions, however, a whole continuum of phases is possible. "Anyon" is a term coined in by Frank Wilczek to describe particles in 2 dimensions that can acquire "any" phase when two or more of them are interchanged. The exchange of two such anyons can be expressed via representations of the braid group and hence, it permits one to encode information in topological features of a system composed of many anyons. Kitaev suggested the possibility that such topological excitations would be stable and could thus be used for robust quantum computation.

This paper aims to

1. give the categorical structure necessary to describe such a computing process;

2. illustrate this structure with a concrete example namely: Fibonacci anyons.

\section{Introduction}

The mathematics and physics of anyons probe the most fundamental principles of quantum mechanics. They involve a fascinating mix of experimental phenomena (the fractional quantum Hall effect), topology (braids), algebra (Temperley-Lieb algebra, braid group and category theory) and quantum field theory. Because of their topological nature, it is hoped that one can use them as stable realisations of qubits for quantum computation, as proposed originally by Kitaev [28]. In this article we review the mathematics of anyons and discuss the relations with braids, topology and modular tensor categories.

The spin-statistics theorem [34, 37, 43, 46] says, roughly speaking, that particles with $\frac{1}{2}$-integer spin satisfy Fermi-Dirac statistics (or are "fermions") while particles with integer spin satisfy Bose-Einstein statistics (or are "bosons"). This statement is one of the few actual theorems of relativistic quantum field theory. What this means is that it can be proved from very general assumptions of quantum mechanics and relativity and does not depend on particular models of particles. 
The proofs traditionally given $[46,17,18]$ involve quantum field theory and rest on assumptions about causality, invariance and positivity of energy. Nevertheless, the statement seems to have a compellingly topological flavor. Indeed such a topological reading has been given by Finkelstein and Rubinstein [20], but for extended objects rather than for elementary particles. The review article by Duck and Sudarshan [17] critiques this and other approaches to the spin-statistics theorem. Their essential point is that elementary particles are not extended objects and thus the topological arguments do not apply. Wightman points out that relativistic invariance is essential to the proof and argues that there is no spin-statistics connection when only euclidean invariance is assumed.

What is clear is that the proof does depend on the fact that relativistic quantum field theory is formulated on a $3+1$ dimensional spacetime. In a $2+1$ dimensional spacetime - which can be realised in the laboratory using surface phenomena - the usual argument for the existence of two kinds of statistics is not valid anymore. Nor is the argument for the existence of two kinds of spins. There is still a connection between spin and statistics but now a continuum of possibilities for each exists.

The experimental investigations of the relevant surface phenomena reveal many surprises. For example, the entities involved have flux tubes that are extended objects, so the topological intuitions underlying the spin-statistics theorem are no longer just heuristic. Furthermore there are collective excitations that behave like particles but like particles with fractional electric charge. Such fractional electric charges are never seen in nature and there are strong theoretical reasons (superselection rules) to think that they cannot occur free in nature.

In the mathematical physics literature, anyons seem to be intimately related to concepts such as modular tensor categories (MTC), a particular class of monoidal categories, modular functors (MF), topological quantum field theory (TQFT) and conformal field theory (CFT). There is, however, an order to this collection of ideas. Indeed, the preceding mathematical constructions may be related in the following way [5]:

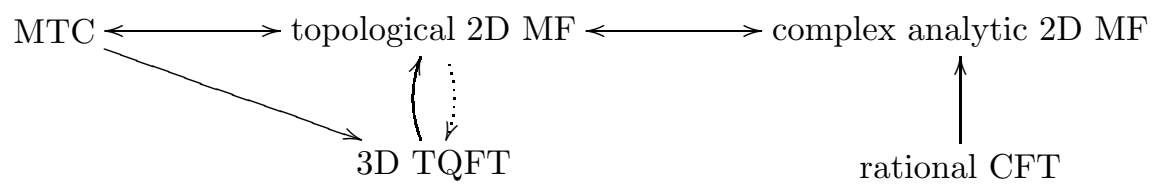

Definitions and an expository account of these relations - along with the precise assumptions needed to define them - are given by Bakalov and Kirilov in [5]. There, they essentially present a complete picture of the results found in $[4,13,27,40,41]$ and [47]. The main point is that most of these structure are essentially equivalent. Using this and the fact that the theory of anyons is correctly described by semisimple MTCs, the categorical semantics 
for topological quantum computation with anyons that we present in section 4 is based on these.

The prerequisites for this paper are relatively modest. We will assume that the reader is familiar with basic category theory and quantum computation. For an introduction to category theory, the reader is referred to [12] or, for a more technical introduction, to [31]. For quantum computation, we suggest [36] or, for a more categorical introduction to the subject [2].

\subsection{Physical background}

The physical effect most associated with anyons is called the fractional quantum Hall effect (fqHe). In order to set the stage we first explain the classical Hall effect, then the (integer) quantum Hall effect and finally the remarkable features of the fqHe. The summary here is very brief and is no substitute for the many thorough papers that have been written in the physics literature. A particularly lucid presentation of the physical ideas appears in the 1998 Nobel lecture of Horst Störmer [45].

The Hall effect was discovered the same year that Einstein was born, 1879. The idea is very simple. Consider a fixed current flowing through a conductor, which we take to be a flat strip. The current flows along the long axis of the strip. If one measures the voltage at various points along the direction of current flow, one gets a drop in the voltage associated with the normal electrical resistance of the material. According to Ohm's law we have $R=V / I$, where $V$ is the measured voltage drop between two points, $I$ is the current and $R$ is the resistance between the two points. One can also measure the voltage drop between two points lying transverse to the flow of the current. One does not expect to see a voltage drop, and indeed none is detected. However, if one applies a magnetic field in the direction perpendicular to the strip then there is a transverse force on the electrons flowing through the conductor: this is the well known Lorentz force law. This can be written as

$$
\mathbf{F}=q \mathbf{v} \times \mathbf{B}
$$

where $\mathbf{F}$ is the force, $\mathbf{v}$ is the velocity of the charged particle, $q$ is its charge and $\mathbf{B}$ is the applied magnetic field. The $\times$ denotes the 3 -vector cross product, so the force is perpendicular to both the direction of motion of the charge and the direction of the applied magnetic field. The upshot is that the electrons are pushed to one side and a voltage develops in the direction across the current flow. This is the Hall effect and is well understood in terms of classical electrodynamics.

The transverse voltage in the Hall effect $V_{H}$, yields an effective Hall "resistance" denoted by $R_{H}=V_{H} / I$. The resistance is now a tensor: the voltage and current are no longer in the same direction. Since the transverse force increases linearly with the applied magnetic field, one expects that $R_{H}$ depends linearly on the applied magnetic field $B$; this is indeed what Hall found. Less 
obvious is the fact that the Hall resistance decreases with increasing electron density. The reason for this is that, for a fixed current, the electrons have to travel faster to achieve the same current so according to the Lorentz force law the transverse force is greater.

A remarkable thing happens in two-dimensional systems at low temperatures. The simple linear behavior of the Hall resistance on the applied magnetic field is replaced by a complicated graph featuring plateaus followed by jumps. Furthermore, the value of the Hall resistance jumps according to a very simple law

$$
R_{H}=\frac{h}{i e^{2}}
$$

where, $h$ is Planck's constant, $e$ is the charge of an electron and $i$ takes on positive integer values. The Hall resistance seems to be "quantised." In addition to this strange behavior of the Hall resistance, the ordinary resistance vanishes at the points corresponding to the plateaus of the Hall resistance. This behavior has been measured very accurately and seems to be universal, i.e. independent of the actual materials used. This phenomenon is called the integer quantum Hall effect. It was discovered in 1980 by Klaus von Klitzing [30].

It is worth emphasising that the two-dimensional nature of the system is crucial. In this case, by "two-dimensional" we mean that electrons are really confined to thin layers and can only move in two dimensions. The "thin" strips used by Hall are, of course, monstrously thick by these standards. Part of the brilliance of the experimentalists who made these discoveries is their skill in making ultra-thin and ultra-pure materials.

Roughly speaking, one can understand the iqHe as arising from the same mechanism that causes electron orbits in atoms to be quantised. From the wave mechanics point of view, electron orbits are quantised because the electron wave function for a confined electron has to obey periodic boundary conditions; this is the same reason that guitar strings have discrete spectra. In the case of the iqHe the Lorentz force does not cause the electrons to move in circular orbits but they tend that way. This is, of course, a very intuitive explanation. A rigorous understanding requires much more sophisticated arguments and detailed calculations. In 1981 Robert Laughlin [32] explained the iqHe as a manifestation of gauge invariance: a deep symmetry principle. However, despite the complexity of the phenomenon, the explanation of the iqHe can be given entirely in terms of the behavior of electrons at low temperature confined to two dimensions and interacting with impurities and with the applied magnetic field.

The fractional quantum Hall effect is like the iqHe except that instead of the integer values appearing in the formula

$$
R_{H}=\frac{h}{i e^{2}}
$$

we can have fractional values of $i$ at which plateaus appear. Furthermore, these fractional values are simple fractions like $\frac{1}{3}$ or $\frac{2}{5}$. This discovery was made by 
Horst Störmer and Daniel Tsui in 1981 and was a complete surprise [45]. The electrons formed complicated composite entities that behaved as if they had fractional charges! Free particles with fractions of the basic electron charge are never seen in nature. It is natural to think that composite entities could seem to have some multiple of the electronic charge but these fractionally charged "particles" were astonishing.

The crucial point is that one cannot explain the fqHe in terms of the behavior of electrons qua electrons. One has to think of the collection of electrons moving in a two-dimensional landscape as collectively forming a fluid with the excitations having a strikingly different character than individual electrons. They are effectively extended objects with nontrivial topological possibilities. The interaction with the magnetic field creates flux tubes that intersect the plane in which the electrons are confined and thus yield ribbonlike objects that can be wound around each other. These are called chargeonfluxon composites.

\section{Spin and Statistics}

Consider a system of $n$ identical particles in quantum mechanics. A permutation of these particles leaves the system physically unchanged in 3 space dimensions. Thus, the only way that the state of the system can change is to be multiplied by a phase. The Hilbert space of the system must carry a unitary representation $\rho$ of the permutation group $S_{n}$.

Now if $\sigma$ is a permutation, $\rho(\sigma)=e^{i \theta} I$ since all that can happen is a change of phase. Let $\tau$ be a transposition: $\tau^{2}=i d$, where $i d$ is the identity permutation. Thus, $\rho\left(\tau^{2}\right)=[\rho(\tau)]^{2}=I$ so $\rho(\tau)= \pm I$. This holds for any transposition. Suppose that $\tau_{1}$ and $\tau_{2}$ share an element, i.e. $\tau_{1}$ interchanges $a$ and $b$ while $\tau_{2}$ interchanges $b$ and $c$. This means that $\tau_{1} \tau_{2} \tau_{1}=\tau_{2} \tau_{1} \tau_{2}$. It follows that $\rho\left(\tau_{1}\right)=\rho\left(\tau_{2}\right)$, for, if one of them, say $\tau_{1}$, mapped to $-I$ and the other to $I$ under $\rho$ we would have $\rho\left(\tau_{1} \tau_{2} \tau_{1}\right)=I$ while $\rho\left(\tau_{2} \tau_{1} \tau_{2}\right)=-I$. We infer that, for a given $\rho$, either all transpositions map to $I$ or all transpositions map to $-I$. Every permutation is the product of transpositions. Thus, there are just two possibilities for $\rho$, either all permutations map to $I$ or all permutations $\sigma$ map to $(-1)^{\operatorname{sign}(\sigma)} I$. Particles obeying the former type of statistics are called bosons and those obeying the latter are called fermions.

We now consider the effect of transporting a particle in a loop. If we bring the particle back to its original position the physics must be unchanged. The Hilbert space must carry a representation of the rotation group $S O(3)$ which describes how the particle transforms under rotation. The group $S O(3)$ can be visualised as a sphere with antipodal points identified. Its homotopy group is $\mathbb{Z}_{2}$. This means that there are two kinds of loops: a closed loop on the surface of the sphere and a curve from a point on the sphere to its antipode. The first kind of loop is deformable to the trivial identity loop: that is, the loop that stays at a point. If one performs a $4 \pi$ rotation the curve is homotopic to the 
identity. Thus a $4 \pi$ rotation must correspond to the identity transformation and a $2 \pi$ rotation to multiplication by \pm 1 .

The group $S O(3)$ has a covering group, that is, a group with trivial homotopy with continuous surjective homomorphism onto $S O(3)$; this is the group $S U(2)$ of unitary 2 -by- 2 matrices with determinant +1 . The representations of $S U(2)$ are well understood: they are classified by a number $s$-called the spin - which is either an integer or a half-integer in natural units. The dimension of the representation is $2 s+1$ : a qubit is often thought of as a spin $\frac{1}{2}$ particle. Particles in nature come in these two species: integer spin or half-integer spin.

According to the spin-statistics theorem, particles that obey Bose-Einstein statistics have integer spin while particles that obey Fermi-Dirac statistics have half-integer spin. This is not an assumption about particular models of elementary particles; it is one of the fundamental theorems of relativistic quantum field theory.

The spin-statistics theorem was first proved by Fierz [19] and Pauli [37] in 1940 for non-interacting quantum fields. Further proofs were given by Pauli himself [38] and deWet [15]. Almost 20 years after the original proof, the spinstatistics theorem was extended to interacting quantum fields by Lüders and Zumino [34] and by Burgoyne [11]. Later several new proofs were given.

The basic mathematical structure of some of the proofs was placed in the context of rigorous quantum field theory by Streater and Wightman [46] using the idea of describing a field theory in terms of the complex analytic properties of vacuum expectation values [48].

The proof in Streater and Wightman [46] is based on the following assumptions:

1. Poincaré invariance,

2. the vacuum is the lowest energy state, thus, the energy spectrum is bounded below,

3. particle annihilation operators annihilate the vacuum,

4. locality: fields at spacelike separation commute or anti-commute and

5. the metric on Hilbert space is positive definite ${ }^{3}$.

The proof given by Streater and Wightman depends heavily on properties of vacuum expectation values of monomials of field operators and their properties as holomorphic functions.

The proof goes by assuming the wrong statistics - e.g. by assuming commutators for spin $\frac{1}{2}$ particles - and then taking vacuum expectation values and finally using analytic continuation to deduce an equation from which it follows that the field vanishes. The analytic continuation process uses a complexified version of the Lorentz group. The essential reason why the proof

\footnotetext{
${ }^{3}$ To a mathematician this is part of the definition of Hilbert space. However, there have been proposals in physics to consider analogues of Hilbert spaces with an indefinite metric. In the physics literature these are sometimes also called "Hilbert spaces."
} 
works is that with the wrong sign in the commutation relations the energy is not bounded below.

This proof makes no mention of topology. Is there a topological reason for the spin-statistics theorem? There have been several papers on this topic: an interesting one is by Finkelstein and Rubinstein [20]. Their argument is based on the idea that the exchange of two particles can be deformed to a rotation by $2 \pi$ of one of them. They give a very appealing heuristic argument for this based on a "rubber band lemma." They make all this precise in the context of solitons - not for the elementary particles for which the original spin-statistics theorem was proved. Rafael Sorkin has been a major presence in the topological approach to spin-statistics theorems. Topological arguments were given by Balachandran et al. [6] for various situations; for example, in [7] they proved a topological spin-statistics theorem for strings. Many subsequent papers were written about a spin-statistics theorem for various kinds of "kinks", "geons" and other entities constructed from topological non-trivialities, see, for example [16]. In an important paper Berry and Robbins [8] showed that one could associate a geometric phase shift associated with exchanging two spin $\frac{1}{2}$ particles and obtain a spin-statistics theorem this way. However, this has not been extended to many particle systems.

There have been several critiques of the non-traditional proofs of the spinstatistics theorem. In a survey article Duck and Sudarshan [17] discuss these and several other proofs. The main point that they make is that the topological proofs apply to "extended objects" and that elementary particles cannot be assumed to have ribbons or other topologically nontrivial structures associated with them. Thus, the topological proofs go not give a substitute for the classical spin-statistics theorem. Wightman [49] pointed out that in the absence of Lorentz invariance there is no spin-statistics connection. Thus attempts to derive it from elementary principles based on euclidean invariance rather than invariance under the Poincaré group are doomed.

\section{Anyons and Braids}

The fact that in two dimensions there are more possibilities for the spin and the statistics is originally due to Leinaas and Myrheim [33], who said "In one and two dimensions a continuum of possible intermediate cases connects the bosons and fermion cases." The possibility was independently rediscovered a few years later by Wilczek [50, 51] and Sorkin [44].

Consider what happens when there are many particles. If they are all labeled as being distinct then arbitrary trajectories can be deformed into the identity transformation. However, in quantum mechanics particles are indistinguishable. Now when there is a trajectory it could correspond to an arbitrary permutation of the particles. However, the strands corresponding to each trajectory can be disentangled so all one has is the permutation. 
When a particle in $2+1$ dimensional spacetime is wound around another twice a nontrivial winding occurs and there is no reason why the phase change should be \pm 1 . For a system of $N$ particles the transformation in the wave function is given by a representation of the braid group.

The state-space of an $n$-particle system has to carry a representation of the braid group rather than of the permutation group. The representations are a much richer collection and we have the possibility of many more kinds of statistics in two dimensions: these particles are the anyons.

There is still a spin-statistics connection, however, it is now more complicated. As we have seen there are more than two possibilities for the "statistics": interchanging particles can cause arbitrary phase shifts. The rotation group in two dimensions is $S O(2)$. This group has the same homotopy group as a circle so it has an infinite family of types of "spin."

There is another new feature to be considered. As we have mentioned before, the physical quasi-particles that arise in the fractional quantum Hall effect are extended objects with charge and tubes of magnetic flux. Not only is there braiding but also twisting. Later, when we formalise the theory categorically we will introduce additional algebraic structure: the aptly named ribbon structure to capture this. For the moment we confine our attention to braiding.

The braid group can be described by giving generators and relations. We think of there being a fixed set of $n$ points along a line segment and we visualise an element of the braid group as a set of strands connecting two such collections of $n$ points. Each strand must go from one of the lower points to one of the upper points. The generators are interchanges of two adjacent strands: this can happen in two ways, the strand of particle $i$ crosses over the strand of particle $i+1$ - we call this $b_{i}$ - or it can cross under, we call this $b_{i}^{-1}$. For $n$ points the generators are $b_{1}$ to $b_{n-1}$ and their inverses. The generators obey the following equations:

$$
\begin{aligned}
b_{i} b_{j}=b_{j} b_{i} & \text { for } \quad|i-j| \geq 2 \\
b_{i} b_{i+1} b_{i}=b_{i+1} b_{i} b_{i+1} & \text { for } 1 \leq i \leq n-1 .
\end{aligned}
$$

which respectively depicts as:

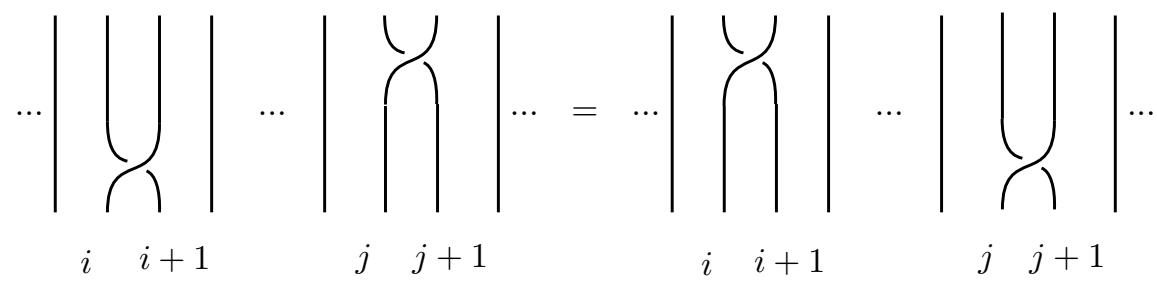

and 


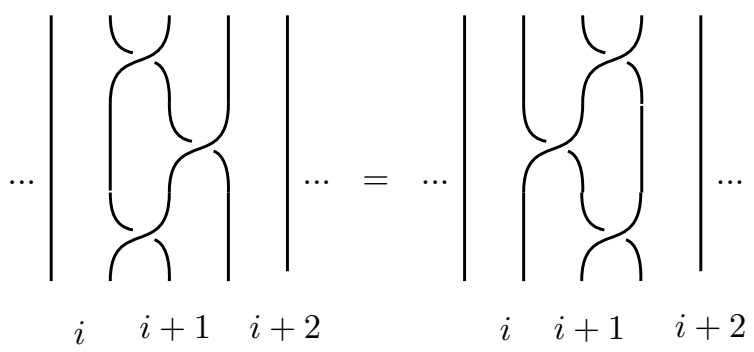

Now a collection of $N$ anyons corresponds to a representation of the braid group on $N$ particles. The simplest case is when they correspond to one-dimensional representations; higher dimensional representations are, in principle, also possible. In the case of one dimensional representations the wavefunction will transform in response to one particle being wound around another by acquiring a phase factor $\exp (i \theta)$; clearly one-dimensional representations of any group are always abelian. Thus, the generator $b_{j}$ is represented by a $\exp \left(i \theta_{j}\right)$. Note that - unlike in the permutation group $-b_{j}^{2} \neq I d$ so $\exp \left(2 i \theta_{j}\right)$ need not be equal to 1 . If one looks at the elements of the braid group in this representation they form an abelian group since, for each generator $b_{j}$ occurring in a word, one gets a factor of $\exp \left(i k \theta_{j}\right)$ where $k$ is the number of times that $b_{j}$ appears in the word minus the number of times $b_{j}^{-1}$ occurs. The order in which the generators appear is not important in this simple representation and for this reason anyons obeying these rules are called abelian anyons. In fact, the equations for the generators (the so-called Yang-Baxter equation) implies that the phase factors are the same for all the generators. To see this note that this equation implies that $\exp \left(i \theta_{j}\right) \exp \left(i \theta_{j+1}\right) \exp \left(i \theta_{j}\right)=\exp \left(i \theta_{j+1}\right) \exp \left(i \theta_{j}\right) \exp \left(i \theta_{j+1}\right)$, whence the result follows immediately. Anyons transforming according to higher dimensional representations of the braid group will not have this simple abelian character: they are called nonabelian anyons.

Physically anyons are collective excitations rather than "elementary" particles. Thus when they are put together they form new excitations in complex ways. What is remarkable is that the complicated physics is summarised by simple algebraic rules called fusion rules. The best way to express the fusion rules is through the theory of semisimple monoidal categories which we will do in the next section.

In order to understand the meaning of fusion rules consider spin in quantum mechanics. Recall that elementary particles carry irreducible representations of $S U(2)$. When two particles are combined to form a composite entity one gets a system that transforms according to the tensor product of the representations. Such a tensor product need not be irreducible so one has to decompose it into irreducible representations: this is called "plethysm" in the mathematics literature. Thus, for example, when one combines a spin $j$ particle with a spin $k$ particle the one gets a system that can be in states of spin $|j-k|$ up to $j+k$ (see, for example, Quantum Mechanics II by A. Messiah [35]). Thus one can write heuristically 


$$
\mathbf{J} \otimes \mathbf{K}=\mathbf{J}-\mathbf{K} \oplus \ldots \oplus \mathbf{J}+\mathbf{K} .
$$

The characterization of the irreducible representation of $S U(2)$ comes from the algebra of the infinitesimal generators (the Lie algebra $\mathfrak{s u}(2)$ ). These are the familiar angular momentum operators obeying the following equations

$$
\left[J_{x}, J_{y}\right] \stackrel{\text { def }}{=} J_{x} J_{y}-J_{y} J_{x}=i \hbar \varepsilon_{x y z} J_{z} .
$$

In general, for a Lie algebra, one can have relations of the form

$$
\left[K_{\alpha}, K_{\beta}\right]=C_{\alpha \beta}^{\gamma} K_{\gamma}
$$

where the $K$ s are from the Lie algebra and the $C$ s are constants called structure constants. For more complicated groups like $S U(3)$ one can write similar relations: in the case of $S U(3)$ there are examples with more than one copy of a given representation.

In considering combinations of anyons of different types we associate with each type of anyon a charge, really this is just another name for "type." Then one will have rules for decomposing combinations of anyons of charges, say, $A$ and $B$, which will take the form $A \otimes B=\ldots$. The theory of monoidal categories is just an abstract presentation of the notion of tensor products so it is the ideal setting to describe the combinatorics of fusion rules.

We can write the fusion rules in a form that looks like the defining equations for a Lie algebra. We write $\langle a, b\rangle$ for the fusion of anyons of type $a$ and $b$. Then we can express fusion rules as

$$
\langle a, b\rangle=N_{a b}^{c} c,
$$

where $a, b$ and $c$ are anyon types and the $N$ s are just natural numbers. Thus a rule of the form

$$
\langle a, b\rangle=2 a+b+3 c
$$

would mean that fusing an $a$-type anyon with a $b$-type anyon yields either an $a$-type anyon, which can occur in two ways, or a $b$ type anyon, or a $c$-type anyon, this last possibility can occur in three ways. For abelian anyons we have $N_{i j}^{k}=0$ unless $k=i+j$, in which case it is 1 . Formally, this looks like the rules for decomposing tensor products of irreducible representations of a Lie group into irreducible representations. However, this does not mean that the fusion rules correspond to the rules for combining irreducible representations of some Lie group. The resemblance is purely formal.

What is the connection between physical anyons and qubits? It is not an anyon by itself that forms a qubit, rather it is the set of fusion possibilities that forms a qubit. If we have a fusion rule with $N_{a b}^{c}=2$ then when we fuse an "a" anyon with a "b" anyon to obtain a "c" anyon, we get a two dimensional space of fusion possibilities. This fusion space forms the qubit. In the case of Fibonacci anyons, to be discussed in detail in later sections, we have two types of anyons $\mathbf{1}$ and $\tau$ with fusion rule $\langle\tau, \tau\rangle=\mathbf{1}+\tau$. If we fuse $\tau$ and $\langle\tau, \tau\rangle$ 
we get $1+2 \cdot \tau$. Thus, if we look at the case when we have a $\tau$ as the result we get a 2-dimensional space of fusion possibilities and this simulates a qubit. An operation or a one-qubit gate on such a qubit consists of a braid connecting three $\tau$ anyons to three $\tau$ anyons where both triple have $\tau$ as global charge. Similarly, a two-qubit gate will be a braiding of two such triples.

\section{The algebra of anyons: Modular tensor categories}

From the discussion so far we can see that there are two aspects of anyonic behavior that need to be formalized. The first is the rather complicated kinematics involving braiding and the second is the dynamics of the anyons. Formalising the former entails having an algebraic structure rich enough to capture charges, braiding and fusion rules and the second requires a way of linking the kinematics with the dynamics according to the usual rules of quantum mechanics formulated in Hilbert spaces.

The kinematic side requires that we have a set of of algebraic rules that describes a system of anyons, their charge, their fusion rules and all this together with an action of the braid group, a formal system which embodies - at least partially - their trajectories in a $2+1$ dimensional space ${ }^{4}$. Our aim is to give a categorical semantics that will take care of both the algebraic structures describing a system of anyons and that allows one to overlay the dynamics on top. Modular tensor categories provides such a language.

At this point it is worth reflecting on the use of categories. The first essential point is that there are different kinds of charges. Thus, an algebraic description must embody several types of objects. This is exactly what categories allow. Indeed, one can think of category theory as a kind of "higherdimensional" algebra as advocated, for example, by John Baez. Second, the paths swept out by anyons are a crucial part of the physical description. One needs an algebraic formalism that allows these relations between anyons to be captured, the morphisms or arrows in a category give the right level of extra structure to express this. In particular we can have notions of objects being isomorphic without being identical.

Consider a category with tensor products, written $\otimes$. It could happen that $A \otimes(B \otimes C)$ is identical to $(A \otimes B) \otimes C$; if this is the case we say we have strict associativity. More commonly we have mere isomorphism between these two objects. In ordinary algebra equations like this are interpreted as identities and one would be forced to make everything strict. With categories one can have these equations holding non-strictly, or, as in the jargon of category theory, "holding up to isomorphism."

Let us first consider the basic properties of anyons and the algebra that is necessary to express these properties:

\footnotetext{
${ }^{4}$ Indeed, the braid group is not sufficient as anyons are extended objects. We need to have ribbons (or framed) strands to adequately represent all movements such as, for instance, a rotation of an anyon by $2 \pi$.
} 
1. First, we have a system of labels, or types, that will represent the charges of our anyons.

2. We also need a way to express a compound system of anyons. This will be expressed by a monoidal (tensor) structure; this way, we will represent a compound system of anyons as the tensor product of their respective charges. The trivial charge is simply the tensor unit. Importantly, this category is not strict monoidal in general. This is physically important because, for instance, the bracketing of a compound system of charges will indicate in which order fusions occur.

3. As we saw in the preceding section, the worldlines of a system of anyons is described by representations of the braid group. We will require that our monoidal category has a braid structure as opposed to being symmetric ${ }^{5}$.

4. We need a way to express the notion of conjugate charge i.e. for a given charge $A$, its conjugate charge $A^{*}$ is the unique charge that can fuse with $A$ to yield the trivial charge. The structure that captures these notions is called a rigid structure.

5. The fact that the objects we are looking at are extended objects - flux tubes - means that, in general, representing their movements graphically with strands in $2+1$ dimensions is not enough; the correct graphical representation is realised by using ribbons, which can be twisted, instead of strands. The algebraic axiomatization of this has been given - long before mathematicians were aware of anyons - and is called a ribbon structure on our category. The axioms for a ribbon structure encapsulate correctly the algebraic rules imposed by the topological properties of ribbons including the possibility of twisting a ribbon.

6 . We need a formal way to express the fusion rules and to map all the preceding algebraic formalism into the context of Hilbert spaces. This will be taken care of by an semisimple structure compatible with all the preceding structures.

7. Finally, we will consider a special class of semisimple ribbon categories called modular tensor categories. Such categories prohibit an infinite number of possible charges for an anyon of a given theory. Moreover, such a category contains within its defining data information about the fusion rules.

Note that most of the results that we present below are known and are discussed in from a physical standpoint in [9, 14, 22, 24] and [39]. Our presentation contrasts with these in the sense that we emphasise the link between the categorical structures and the physical phenomena.

We now give detailed definitions of the categorical structures we mentioned above. Some of them are already given in [12] where the reader is referred for a more detailed discussion on these topics.

${ }^{5}$ Being symmetric means that the braiding $\sigma$ is such that $\sigma_{B, A} \sigma_{A, B}=1_{A \otimes B}$ for all $A$ and $B$. 


\subsection{Charges and compound systems: Monoidal categories}

Definition 1. A monoidal category is a category $\mathbf{C}$ equipped with a bifunctor

$$
\otimes: \mathbf{C} \times \mathbf{C} \rightarrow \mathbf{C}
$$

and three natural isomorphisms $\alpha, \lambda$ and $\rho$ with components

$\alpha_{A, B ; C}:(A \otimes B) \otimes C \rightarrow A \otimes(B \otimes C), \quad \lambda_{A}: \mathbf{1} \otimes A \rightarrow A$ and $\rho_{A}: A \otimes \mathbf{1} \rightarrow A$

such that for all $A, B, C$ and $D \in|\mathbf{C}|$, both

(i) Pentagon axiom.

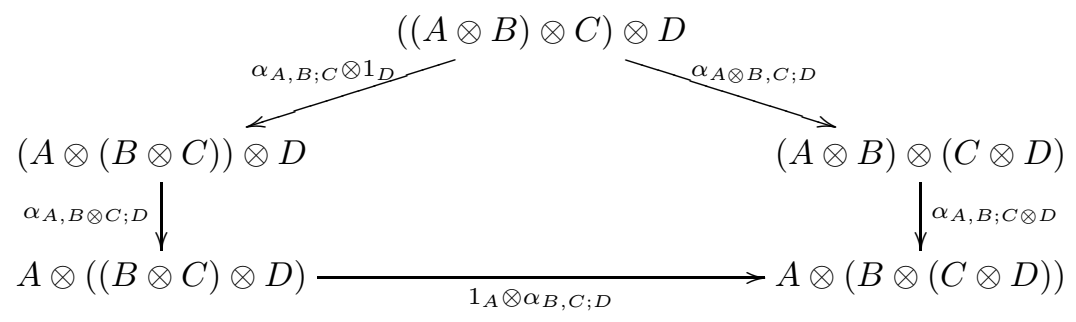

and

(ii) Triangle axiom.

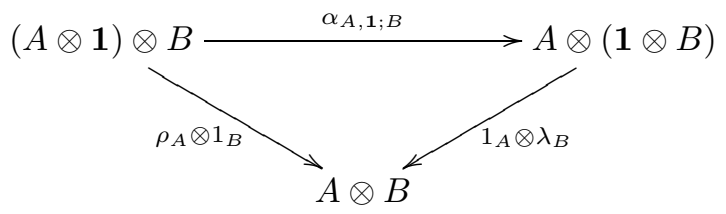

commute.

We interpret the components of this definition as follows:

- Objects: We will regard an $A \in|\mathbf{C}|$ as a label for a set of anyons. Note that this set might contain a single anyon. However, in that case, the object must satisfy some properties that we will consider below. Nonetheless, for simplicity purpose, in what follows, we will assume that an object $A$ is the charge of a single anyon; this will make the explanations simpler.

- Tensor: Given a set of $n$ charges $A_{1}, A_{2}, \ldots, A_{n}$, the compound charge of the system will be described by the $n$-fold tensor product $A_{1} \otimes A_{2} \otimes \ldots \otimes A_{n}$.

- Unit: The unit $\mathbf{1} \in|\mathbf{C}|$ is the label indicating the trivial charge.

The natural isomorphisms are interpreted as:

- $\quad \alpha$ : The bracketing of an $n$-fold tensor product indicates the order of the fusions of the $n$ components of the tensor product. The associativity isomorphism is used to change the pattern of fusion meaning that

$$
\alpha_{A, B ; C}:(A \otimes B) \otimes C \rightarrow A \otimes(B \otimes C)
$$

changes the order of the fusions from $A \otimes B$ to $B \otimes C$ occurring first. 
- $\lambda$ and $\rho$ : The natural isomorphisms $\lambda_{A}: \mathbf{1} \otimes A \rightarrow A$ and $\rho_{A}: A \otimes \mathbf{1} \rightarrow A$ simply tells us that combining an anyon with charge $A$ with the trivial charge 1 changes nothing about the compound charge or even to the charge of an anyon obtained by fusing these two anyons together.

\subsection{Worldlines: Braided monoidal categories}

To correctly handle the movements of the anyons, our category needs at least a braid structure. As a compound system of charges is represented by their tensor product, the braiding will act on the components of such a tensor product of charges. Thus, the braid structure must behave coherently with the monoidal structure. What we need is called a braided monoidal category which is formally defined as follows:

Definition 2. $A$ braided monoidal category $\mathbf{C}$ is a monoidal category equipped with a family of isomorphisms

$$
\sigma_{A, B}: A \otimes B \stackrel{\sim}{\longrightarrow} B \otimes A
$$

natural in $A$ and $B \in|\mathbf{C}|$, such that

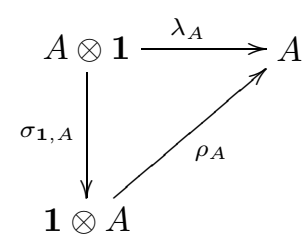

and both

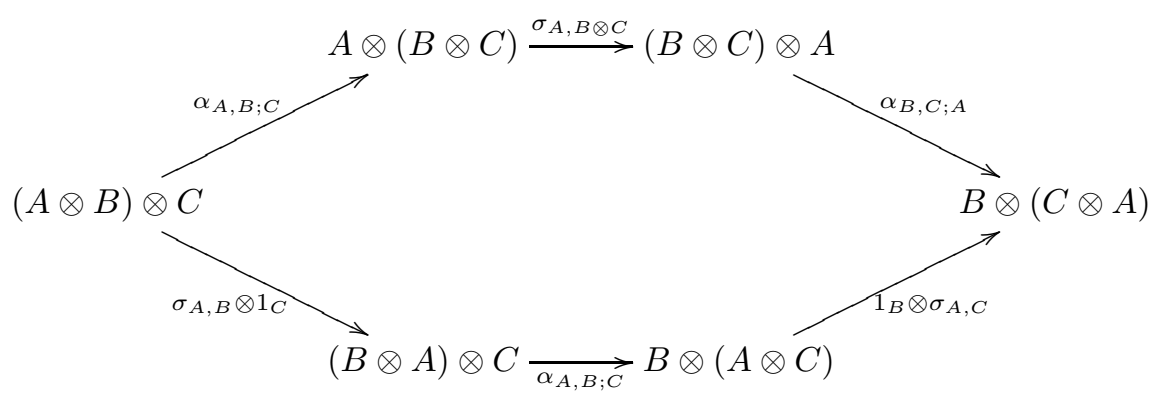

and the same diagram with $\sigma^{-1}$ instead of $\sigma$, commute for all $A, B$ and $C \in$ $|\mathbf{C}|$.

Of course,

- $\sigma$ : The natural isomorphism $\sigma_{A, B}$ is interpreted as the exchange of the charges $A$ and $B$. 
The reader might wonder if such a definition is enough for our purposes, in the sense that this is enough to adequately express the worldlines of a tuple of anyons. The remarkable answer is "yes!" There is a coherence theorem for braided monoidal categories, due to Joyal and Street, which says that given a natural isomorphism built from tensoring and composing identities and components of $\alpha, \lambda, \rho$ and $\sigma$ from the braided monoidal structure, there is an associated braid. Moreover, two such isomorphisms are equal if and only if their associated braids are equal. The proof of this theorem is quite technical and, for our purposes, the preceding comment is enough. We refer the reader to [25] for the details.

Remark 1. Despite the fact that the our category is not strict and since every monoidal category is equivalent to a strict monoidal category [31], we may omit bracketing and unit isomorphisms if these aren't necessary for the exposition.

\subsection{Charge conjugation: Rigidity}

Now that we have some of the algebraic tools that describe the worldlines of anyons, we introduce another structure to express the conjugation of the charges which is similar to the notion of a compact structure on a monoidal category. This is given via the notion of duals within $\mathbf{C}$.

Definition 3. Let $\mathbf{C}$ be a braided monoidal category and $A \in|\mathbf{C}|$. A dual of $A$ is an object $A^{*} \in|\mathbf{C}|$ together with two morphisms $i_{A}: \mathbf{1} \rightarrow A \otimes A^{*}$ and $e_{A}: A^{*} \otimes A \rightarrow \mathbf{1}$ that are such that

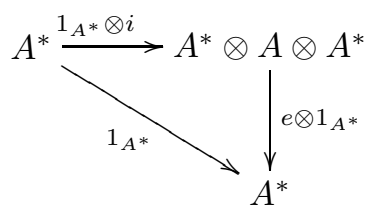

and

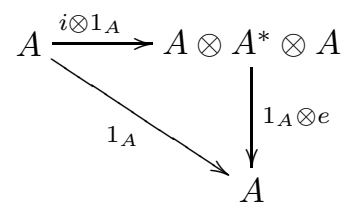

commute. A braided monoidal category $\mathbf{C}$ is rigid if each $A \in|\mathbf{C}|$ has a dual.

Physically speaking, we will interpret the structural morphisms of the previous definition in the following way:

- $\quad i$ : is interpreted as the creation of a particle-antiparticle pair - anyons with respective dual charges - and

- $\quad e$ : as the annihilation of an antiparticle-particle pair.

Now, given an $f: A \rightarrow B$ in a rigid braided monoidal category, we can define $f^{*}: B^{*} \rightarrow A^{*}$ as the composite

$$
B^{*} \stackrel{1_{B^{*} \otimes i_{A}}}{\longrightarrow} B^{*} \otimes A \otimes A^{*} \stackrel{1_{B^{*} \otimes f \otimes 1} A^{*}}{\longrightarrow} B^{*} \otimes B \otimes A^{*} \stackrel{e_{B} \otimes 1_{A}}{\longrightarrow} A^{*} .
$$

It is easily verified this operation on morphisms together with $A \mapsto A^{*}$ on objects defines a functor.

Later, we will need the following 
Proposition 1. [5] Let $\mathbf{C}$ be a rigid braided monoidal category and $B \in|\mathbf{C}|$ together with its dual $B^{*} \in|\mathbf{C}|$ then, there are canonical isomorphisms

$$
\begin{aligned}
& \operatorname{Hom}(A \otimes B, C) \simeq \operatorname{Hom}\left(A, C \otimes B^{*}\right) \\
& \operatorname{Hom}(A, B \otimes C) \simeq \operatorname{Hom}\left(B^{*} \otimes A, C\right) .
\end{aligned}
$$

\subsection{Graphical calculus for rigid braided monoidal categories}

As we showed in section 3, we can illustrate components of the braid group and their composition with pictures. In fact, we can do more: we will now give pictorial representation depicting completely the trajectories of anyons in $2+1$ dimensions. Such a graphical calculus comes for free with rigid braided monoidal categories and adequately represents morphisms in such categories [5].

Let us now give the basic building blocs of such a graphical calculus:

- The identity on $\mathbf{1} \in|\mathbf{C}|$ is represented as the empty picture. This is not surprising: adding the trivial charge to the system is the same thing as adding nothing.

- The identity on a charge $A \in|\mathbf{C}|$ and its dual are respectively represented by

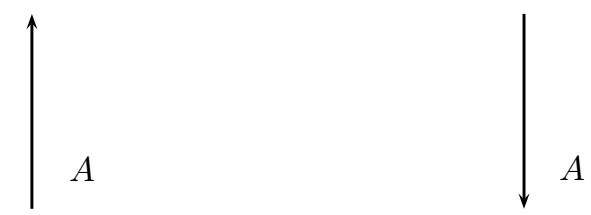

- A morphism $f: A \rightarrow B$ is depicted as

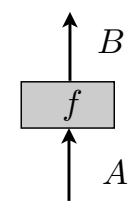

- The composition of morphisms $f: A \rightarrow B$ and $g: B \rightarrow C$ is given by stacking the graphical representations of and $f$ and $g$ and connecting the arrows labeled by $B$ i.e.,

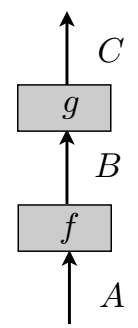


- The tensor product of morphisms $f: A \rightarrow B$ and $g: C \rightarrow D$ is given by aligning the graphical representations of and $f$ and $g$ side by side in the $f \otimes g$ order i.e.,

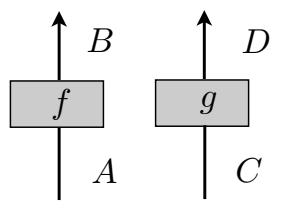

hence obtaining the representation for $f \otimes g: A \otimes C \rightarrow B \otimes D$.

- The morphisms $i_{A}: \mathbf{1} \rightarrow A \otimes A^{*}$ and $e_{A}: A^{*} \otimes A \rightarrow \mathbf{1}$ of the rigid structure on $A \in|\mathbf{C}|$ are represented as

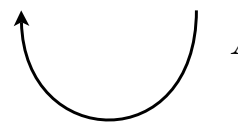

and

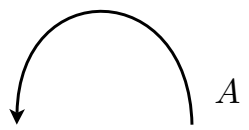

respectively.

- The braiding $\sigma_{A B}: A \otimes B \rightarrow B \otimes A$ and it's inverse are respectively depicted as

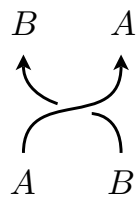

and

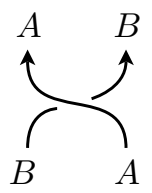

Remark 2. Note that the natural isomorphisms $\alpha, \rho$ and $\lambda$ are not captured by this formalism. For the first, we will introduce a graphical notation for fusion later. For the latter two, it does not matter as already mentioned in our comment on the representation of $1_{1}$.

\subsection{A twist in the worldlines: Ribbon categories}

As noted in the introduction of this section, the braid group is not enough to capture completely the kinematics of the anyons. For instance, an anyon can revolve around some center by $2 \pi$ and the change induced on the system is not an identity. Let us consider what this means.

Based on the language we already have from the theory of rigid braided monoidal categories, we can build the following process:

1. A pair of particles with respective charges $A$ and $A^{*}$ is created,

2. The two particles are swapped with the particle of charge $A$ going behind the antiparticle of charge $A^{*}$ and, finally,

3. They annihilate. 
Such a process is built from structural morphisms as

$$
f=i_{A} \circ \sigma_{A, A^{*}}^{-1} \circ e_{A} .
$$

From the graphical calculus on morphisms, $f: \mathbf{1} \rightarrow \mathbf{1}$ gets depicted as:

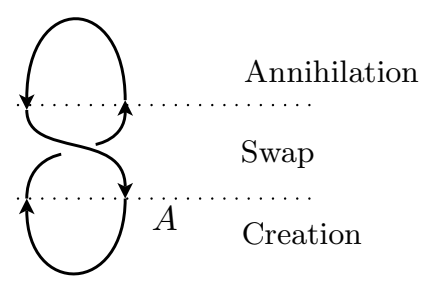

The key point here is that the amplitude of the process is non-trivial as there is an exchange occurring. Moreover, we have the following topological equivalences:

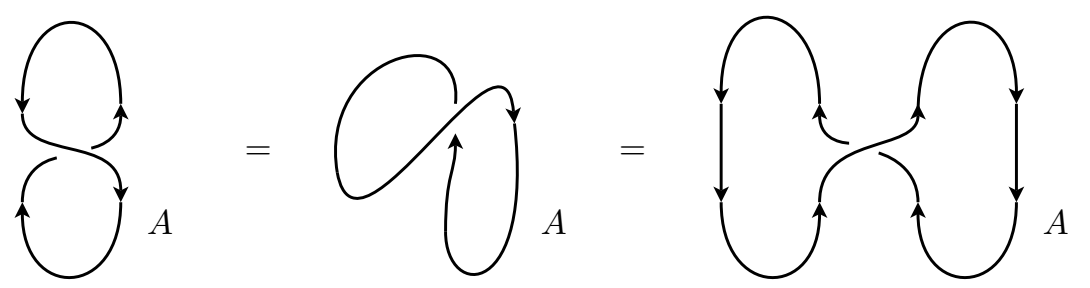

telling us that the amplitude for the first process is equal to the amplitude of the third which is, from bottom to top:

1. The creation of both an antiparticle-particle and particle-antiparticle pair,

2. The exchange of the particle from the left pair with the one from the right and

3. The annihilation of the particle from the right pair with the antiparticle from the left pair and the annihilation of the particle from the left pair with the antiparticle the right pair.

Now, consider the following deformation:

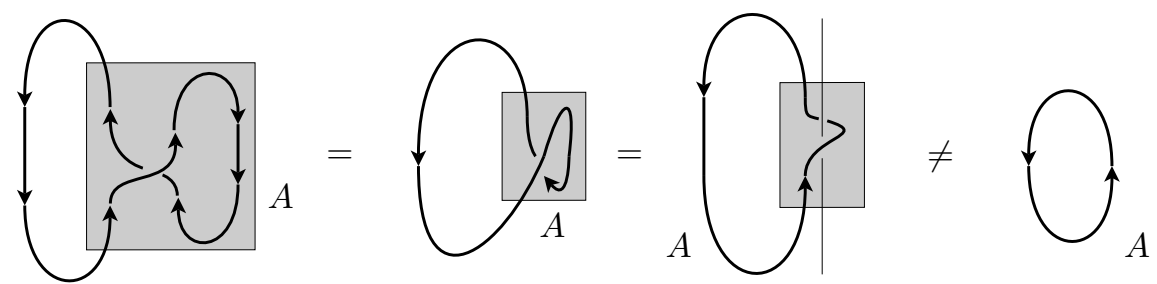

The third picture can be read as the creation of an antiparticle-particle pair and the particle gets rotated about $2 \pi$ of some center which illustrated in the gray box. Now, as noted, this amplitude is different from the trivial amplitude 
depicted as the fourth picture. We conclude from this that illustrating the worldlines of our anyons with strands is not completely faithful to the process. Instead, we will use ribbons - or framed strands - so that:

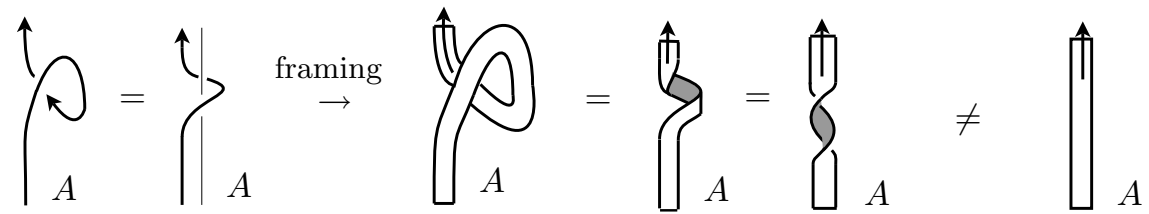

This "winding around some center" is almost given already by the rigid braided monoidal structure. Indeed, in any rigid braided monoidal category $\mathbf{C}$, one can define

$$
\gamma_{A}: A^{* *} \rightarrow A \quad \text { as } \quad \gamma_{A}:=\left(A \otimes e_{A^{*}}\right) \circ\left(A \otimes \sigma_{A^{*} A^{* *}}^{-1}\right) \circ\left(i_{A} \otimes A^{* *}\right)
$$

for any $A \in|\mathbf{C}|$ (the reader may check that $\gamma_{A}$ is topologically equivalent to the framed ribbon of the preceding picture). However, note that we have a type mismatch if we compare with the twist depicted above as the later is of type $A \rightarrow A$. To complete the definition, we need a natural isomorphism of type $A \rightarrow A^{* *}$ which will behave coherently with the rest of the structure. Formally:

Definition 4. A ribbon category ${ }^{6} \mathbf{C}$ is a rigid braided monoidal equipped with a natural isomorphism $\delta$ with components

$$
\delta_{A}: A \rightarrow A^{* *}
$$

satisfying

i) $\delta_{A \otimes B}=\delta_{A} \otimes \delta_{B}$;

ii) $\delta_{A^{*}}=\left(\delta_{A}^{*}\right)^{-1}$ and,

iii) $\delta_{\mathbf{1}}=1$.

This is enough to formally define the "twist" that we discussed:

Definition 5. Let $\mathbf{C}$ be a ribbon category. The twist map is defined as the natural isomorphism $\theta$ with components

$$
\theta_{A}:=\gamma_{A} \circ \delta_{A}: A \rightarrow A
$$

Graphically, this is denoted as

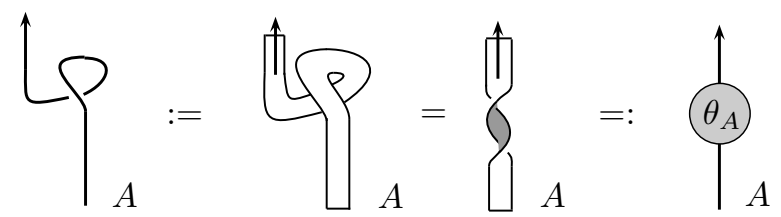

\footnotetext{
${ }^{6}$ Sometimes called a tortile category.
} 
where we have deliberately omitted the isomorphism $\delta_{A}$ to avoid cluttering the picture. Note that we can (and did) get back to our strand notation and this is done without loss inasmuch as we use this notation for the twist, keeping in mind that this is a rotation of $2 \pi$ of the strand around its center.

Interestingly, provided that we use these graphical conventions, any two processes that can obtained by obtained by continuously deforming one into the other will have the same amplitude! In fact, a theorem due to Reshetikhin and Tuarev tells us that for two such diagrams, the isomorphisms corresponding to each of these diagrams are equal. For an exact statement and a detailed proof, the reader is referred to [40].

This completes our discussion on the algebraic context describing the worldlines of anyons.

\subsection{Towards fusion: Semisimple ribbon categories}

Now, we need fusion rules to build the fusion spaces where our quantum computational interpretation will take place but before, we need to introduce some new concepts:

Definition 6. $A$ morphism $m: A \rightarrow B$ is a monomorphism (or is monic) when for any two $f, g: C \rightarrow A$, we have

$$
m \circ f=m \circ g \quad \Rightarrow \quad f=g .
$$

Conversely,

Definition 7. A morphism $h: A \rightarrow B$ is an epimorphism (or is an epi) when for any two $f, g: B \rightarrow C$,

$$
f \circ h=g \circ h \quad \Rightarrow \quad f=g .
$$

Because of its defining condition, a monomorphism (resp. epimorphism) is sometime called left-cancellable (resp. right-cancellable). The two previous definitions generalise the notions of injection and surjection in the usual sense. In fact, one may check that in Set, these concepts coincide i.e., monics are exactly injections and epis are exactly surjections.

Definition 8. A zero object in a category $\mathbf{C}$ is an object $0 \in|\mathbf{C}|$ which is both initial and terminal.

In particular, the presence of such an object enables us to define a zero morphism. Indeed, if $\mathbf{C}$ is a category with a zero object then, for any $A, B \in|\mathbf{C}|$, there exists a unique morphism $0: A \rightarrow B$ defined as the composition $A \rightarrow 0 \rightarrow B$. Uniqueness of the zero morphism follows from the fact that 0 is simultaneously initial and terminal and hence, the set of arrows to and from it are singletons.

Such an object and its associated morphism enables in turn a generalisation of the notion of kernel as follows: 
Definition 9. The kernel of a morphism $f: A \rightarrow B$ in $\mathbf{C}$, a category with a zero object, is an arrow

$$
\operatorname{Ker}(f):=k: S \rightarrow A
$$

such that if $f \circ k=0$, the zero morphism, then for every $h: C \rightarrow A$ such that $h \circ f=0, h$ factors uniquely through $k$ as $h=h^{\prime} \circ k$. Diagrammatically,

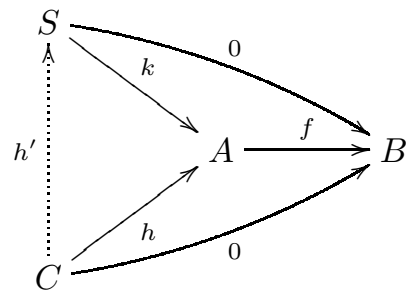

We can also define the dual notion:

Definition 10. The cokernel of a morphism $f: A \rightarrow B$ in $\mathbf{C}$, a category with a zero object, is an arrow

$$
\operatorname{CoKer}(f):=u: B \rightarrow S
$$

such that if $u \circ f=0$ and if $h: B \rightarrow C$ is such that $h \circ f=0$, then $h$ factors uniquely through $u$ as $h=u \circ h^{\prime}$ i.e.:

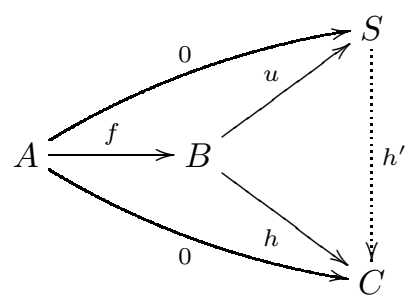

We now can state the central definition of this subsection ${ }^{7}$ :

Definition 11. A category $\mathbf{C}$ is

a. Preadditive if its homsets are abelian groups (written additively) and the composition of morphism is bilinear over the integers;

b. It is additive if, in addition, every finite set of objects has a biproduct;

c. It is preabelian if it is additive and every morphism in $\mathbf{C}$ has a kernel and a cokernel and finally,

d. It is abelian if, in addition, every monomorphism is a kernel and epimorphism is a cokernel.

7 This definition is equivalent [23] to the standard definition of an abelian category [31]. 
Let us emphasise the most important part of the previous definition from our point of view. The fact that a category $\mathbf{C}$ is abelian comes with a bonus: indeed, the previous definitions not only says that the kernels and the cokernels in $\mathbf{C}$ behave the same way as in vector spaces but also that its hom-sets are abelian groups and this can be seen as a first step toward an interpretation of our formalism within the context of complex vector spaces. To really get there, we need yet another notion inspired by the following fact: the charges of our basic anyons are irreducible in the sense that they cannot be decomposed into more elementary entities ${ }^{8}$. Such a property of the charges can be recast in categorical terms as follows:

Definition 12. Let $\mathbf{C}$ be an abelian category then, an $S \in|\mathbf{C}|$ such that $S \neq 0$ is simple if for all $B \in|\mathbf{C}|, f: B \hookrightarrow S$ is either the zero morphism or an isomorphism.

This is the same as saying that $A$ has no other subobject other than 0 and itself. From this, we have

Definition 13. An abelian category $\mathbf{C}$ is semisimple if any $A \in|\mathbf{C}|$ is such that

$$
A \simeq \bigoplus_{j \in J} N_{j} S_{j}
$$

where $S_{j}$ is a simple object, $J$ is the set of isomorphism classes of simple objects and $N_{j} \in \mathbb{N}$ are such that only a finite number of them are non-zero.

This is enough to give our last definition in which there are now two distinct monoidal products; one from the abelian structure written as $\oplus$ and one from the ribbon structure denoted by $\otimes$.

Definition 14. A semisimple ribbon category $\mathbf{C}$ is a semisimple category endowed with a ribbon structure where the tensor unit $\mathbf{1} \in|\mathbf{C}|$ is simple, the tensor product is bilinear and where for each simple object $S \in|\mathbf{C}|, \operatorname{End}(S) \simeq \mathbb{K}$, a field of characteristic 0 .

Remark 3. In what follows, we will assume that the field mentioned in the last definition is $\mathbb{C}$, the complex field.

Remark 4. To lighten the notation, we will often use the index of simple objects to identify morphisms involving these. For instance, $1_{i}$ is the identity on the simple object $S_{i}$ and the natural isomorphism $\sigma_{i j}$ is of type $S_{i} \otimes S_{j} \rightarrow S_{j} \otimes S_{i}$. Correspondingly, we will label the wires in the picture calculus only with the index $i$ instead of the label $S_{i}$.

Now, having such a semisimple structure on $\mathbf{C}$ has many consequences. First, we can now handle fusions of anyons. Second, it is from this structure that complex vector spaces arise. Indeed, it is this fact that will enables us to define splitting spaces and unitary representations of the braid group therein. Such a structure also enables the following results:

\footnotetext{
${ }^{8}$ Of course, this is an approximation in the effective field theory of these excitations.
} 
Proposition 2. In semisimple ribbon categories, there are natural isomorphisms

$A \otimes(B \oplus C) \simeq(A \otimes B) \oplus(A \otimes C)$ and $(A \oplus B) \otimes C \simeq(A \otimes C) \oplus(B \otimes C)$.

Moreover,

$$
(A \oplus B)^{*} \simeq A^{*} \oplus B^{*} \text { and } 0^{*}=0 .
$$

\subsection{Modular tensor categories}

Semi-simple ribbon categories can already handle the kinematics of a system of anyons, our description of a particular model of anyon will take place within a particular class of such categories namely, modular tensor categories. First, such categories only allow a finite number of charges or rather, a finite set of isomorphism classes of simple objects. There is no reason to think that this is true in nature, however, all the applications that we have in mind for quantum computation can be done with a finite number of anyon types. A second point that we will inspect later is that the $s$-matrix defining the modularity condition has surprising connexions with the fusion rules.

More generally, these extra assumptions on semisimple ribbon categories lead to many interesting results; in particular, we can derive an equivalence between MTCs and modular functors. Since the goal of this paper is to give an introduction to computing with anyons, these results are not pursued here. The interested reader might want to look at [5] for a complete exposition of these subjects.

Definition 15. A modular tensor category is a semisimple ribbon category $\mathbf{C}$ such that

1. Finiteness condition: The index set $J$ of isomorphism classes of simple objects is finite and $S_{0}:=\mathbf{1}$.

2. Modularity condition: For $i, j \in J$, the matrix $s$ with entries

$$
(s)_{i j}=\left[\lambda_{\mathbf{1}} \circ\left(e_{i} \otimes e_{j}\right) \circ\left(i d_{i} \otimes \sigma_{i j} \otimes i d_{j}\right) \circ\left(i d_{i} \otimes \sigma_{j i} \otimes i d_{j}\right) \circ\left(i_{i} \otimes i_{j}\right) \circ \lambda_{\mathbf{1}}^{-1}\right]_{i j}
$$

which are depicted as

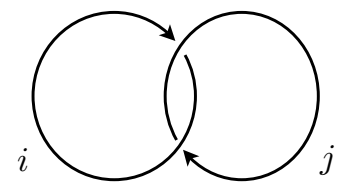

is invertible.

Remark 5. We indeed get a matrix for $s$ since we have $\operatorname{End}(\mathbf{1}) \simeq \mathbb{C}-$ a field of characteristic 0 - and the morphism depicted above is really of type $\mathbf{1} \rightarrow \mathbf{1}$ hence, a scalar. 
The scalar components of the $s$-matrix form the so-called Hopf link. They can be thought of as the amplitude of the following process:

1. Two pairs of particle-antiparticle are created,

2. The particle from one pair is wound around the particle from the other pair and

3. The two pairs annihilate.

We don't have yet the machinery to describe the surprising results that the modularity constraint entails, but we will do so in the subsection on the Verlinde Formula below.

\subsection{Fusion rules}

For now on, we fix $\mathbf{C}$ to be a modular tensor category. As already mentioned, the charge of an anyon is represented by a simple object in C. Suppose that two anyons with charges $S_{i}$ and $S_{j}$ fuse together into an anyon of charge $S_{k}$ and that in $N_{i j}^{k}$ ways, we will write this as

$$
S_{i} \otimes S_{j} \simeq N_{i j}^{k} S_{k}
$$

There, the lower labels of $N_{i j}^{k}$ then indicate which charges fuse together in order to yield the charge identified by the upper label.

Remark 6 . Note that such an expression always makes sense, since by assumption the category is semisimple thus, each object is isomorphic to a direct sum of simple ones.

Now, the fusion process can produce different charges and this constitutes a generalisation of our description above. Taking in account this fact, we get the following

Definition 16. [Fusion rule] Let $S_{i}$ and $S_{j}$ be simple objects in $\mathbf{C}$ and $J$ be the index set for the isomorphism classes of simple objects. The fusion rule of $S_{i}$ and $S_{j}$ is given by

$$
S_{i} \otimes S_{j} \simeq \bigoplus_{k \in J} N_{i j}^{k} S_{k}
$$

There, the coefficients $N_{i j}^{k}=\operatorname{Dim}\left(\operatorname{Hom}\left(\left(S_{i} \otimes S_{j}\right), S_{k}\right)\right.$ are called the fusion coefficients of the fusion rule.

It is easy to verify that the fusion coefficients satisfy

$$
N_{i j}^{k}=N_{j i}^{k}=N_{j k^{*}}^{i^{*}}=N_{i^{*} j^{*}}^{k^{*}}=N_{i^{*} j^{*} k}^{0}=\ldots \text { and } N_{i j}^{0}=\delta_{i j^{*}} .
$$

and hence, in particular, we can lower and raise indices. 


\subsection{Translation to the Hilbert space context: Fusion and splitting spaces}

We now come to the crux of the story. We now need to connect our rather abstract algebraic rules describing the kinematics of our anyons to dynamics which is expressed in the language of Hilbert spaces. Such a translation is already built-in a modular tensor category $\mathbf{C}$. Indeed, we will use the facts that hom-sets of $\mathbf{C}$ are vector spaces over $\mathbb{C}$ and that for all simple objects $S$, $\operatorname{End}(S) \simeq \mathbb{C}$ to build the so-called splitting and fusion spaces in which we will simulate our qubits and coqubits.

Before carrying on to define such spaces for a family of anyons, we will need the following variant of Schur's lemma:

Lemma 1. Let $\mathbf{C}$ be a semisimple abelian category and $S_{i}, S_{j} \in|\mathbf{C}|$ be simple objects such that $i \neq j$, then $\operatorname{Hom}\left(S_{i}, S_{j}\right)=\{0\}$.

Proof: Let $f: S_{i} \rightarrow S_{j}$ be arbitrary and consider $\operatorname{Ker}(f): U \hookrightarrow S_{i}$ (the kernel is necessary monic) then, as $S_{i}$ is simple by assumption, it follows that $\operatorname{Ker}(f)$ is either 0 or an isomorphism. If the kernel is 0 , then $f$ is an injection and hence, an isomorphism since $S_{j}$ is simple, but this cannot be possible as $i \neq j$. Therefore, by simplicity of $S_{i}$ we have $U \simeq S_{i}$ and $f=0$ from which we conclude that $\operatorname{Hom}\left(S_{i}, S_{j}\right)=\{0\}$ as $f$ is arbitrary.

As a consequence of semisimplicity, the homset

$$
\operatorname{Hom}\left(S_{k}, S_{i} \otimes S_{j}\right)
$$

is really a complex vector space whose dimension is fixed by the fusion rule. Generally speaking, the passage from our modular tensor category to the category of finite dimensional complex vector spaces will be handled by this fact.

Definition 17. Let $S_{i}, S_{j}$ and $S_{k}$ be simple objects in $\mathbf{C}$, a splitting space ${ }^{9}$ is a complex vector space

$$
V_{k}^{i j} \simeq \operatorname{Hom}\left(S_{k}, S_{i} \otimes S_{j}\right)
$$

of dimension $N_{k}^{i j}=\operatorname{dim}\left(\operatorname{Hom}\left(S_{k}, S_{i} \otimes S_{j}\right)\right)$ - the number of ways the charge $S_{k}$ can split as the compound charge $S_{i} \otimes S_{j}$. Its states

$$
\operatorname{Hom}\left(S_{k}, S_{k}\right) \longrightarrow \operatorname{Hom}\left(S_{k}, S_{i} \otimes S_{j}\right)
$$

are called splitting states.

\footnotetext{
${ }^{9}$ Such a space is also called a fusion space however, as the initialisation of a state takes place via a splitting, we prefer our proposed terminology.
} 
Note that the type of the splitting states makes sense. Indeed, if the splitting rule tells us that $\operatorname{Dim}\left(\operatorname{Hom}\left(S_{k}, S_{i} \otimes S_{j}\right)\right)=N_{k}^{i j}$, then through the fact homsets are complex vector spaces, a splitting state is a linear map of type

$$
\mathbb{C} \longrightarrow \mathbb{C}^{N_{k}^{i j}}
$$

We now define the basis vectors of a splitting space. Let us introduce the following notation: For $S_{i}, S_{j}$ and $S_{k}$, some simple objects of $\mathbf{C}$, if the following fusion rule $S_{i} \otimes S_{j} \simeq N_{i j}^{k} S_{k}$ holds, we will denote the basis state representing the corresponding to the $\eta^{\text {th }}$ possible splitting of $S_{k}$ into $S_{i} \otimes S_{j}$ as $|i j ; k, \eta\rangle$ so that the set of basis vectors that spans the splitting space $V_{k}^{i j}$ is

$$
\left\{|i j ; k, \eta\rangle \mid \eta \in\left\{1,2, \ldots, N_{k}^{i j}\right\}\right\} .
$$

In the category FdVect $_{\mathbb{C}}$, the basis vector $|i-1\rangle: \mathbb{C} \rightarrow \mathbb{C}^{n}$ of $\mathbb{C}^{n}:=\mathbb{C} \oplus \ldots \oplus \mathbb{C}$ $n$ times, can be assimilated to the $i$-th canonical injection

$$
\iota_{i}: \mathbb{C} \longrightarrow \mathbb{C}^{n} \quad:: \quad 1 \quad \mapsto\left(\begin{array}{c}
0 \\
\vdots \\
1 \\
\vdots \\
0
\end{array}\right)
$$

with the " 1 " on the $i$-th line and 0 's elsewhere. Lifting this to our context, we can define the basis vector

$$
|i j ; k, \eta\rangle
$$

for fixed $k$ and $\eta$ as

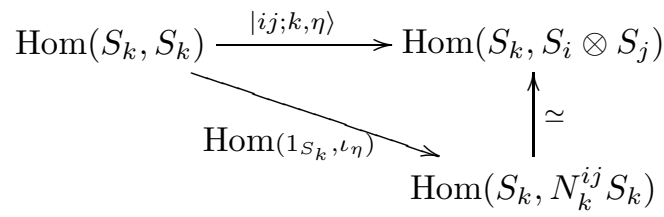

where $\iota_{\eta}$ is the $\eta^{\text {th }}$ canonical injection into the $N_{k}^{i j}$-fold biproduct of $S_{k}$ 's.

According to the general form of the fusion rule, the compound charge $S_{i} \otimes S_{j}$ could be obtained via the splitting of different charges. In the light of lemma 1, each such splitting spaces are orthogonal one to the other. Thus, what we actually get is a tuple ${ }^{10}$ of splitting spaces $\left\langle V_{k_{1}}^{i j}, \ldots, V_{k_{n}}^{i j}\right\rangle$ where $\left\{k_{1}, \ldots, k_{n}\right\} \subseteq J$, the index set of isomorphism classes of simple objects. Each component of this tuple carries a different possible branch of the computation labeled by the charge $S_{k_{l}}$. These spaces are mutually orthogonal in the

${ }^{10}$ Strictly speaking, it is a biproduct of fusion spaces but as the later is simultaneously a product and a coproduct, it also makes sense to speak of tuples. 
topological vector space $V^{i j}$ of dimension $\sum_{k_{l} \in J} N_{k_{l}}^{i j}$ spanned by the set of vectors

$$
\left\{\left|i j ; k_{l}, \eta\right\rangle \mid k_{l} \in\left\{k_{1}, \ldots, k_{n}\right\}, \eta \in\left\{1,2, \ldots, N_{k_{l}}^{i j}\right\}\right\} .
$$

Remark 7. We can also define costates. Indeed, so far, we have spoken of a splitting state but the anyons can also - of course - fuse together thus yielding costates. As an example, for each basis vector $|i j ; k, \eta\rangle$, we can define a bra $\langle i j ; k, \eta|$ dualising the defining diagram for the basis vector and using canonical projections instead of the canonical injections.

Returning to our splitting spaces, we see that there is a morphism of type $S_{k} \rightarrow S_{i} \otimes S_{j}$ underlying each basis vector of a given splitting space. As it is determined by the $\eta^{\text {th }}$ canonical injection and the splitting rule, we depict such a morphism as:

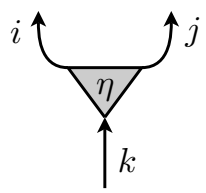

Of course, such a family of morphisms satisfies the following two relations:

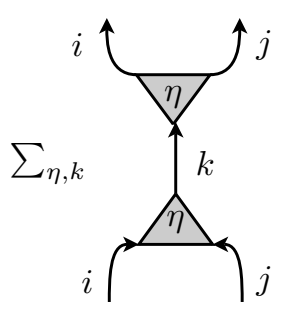

(a)

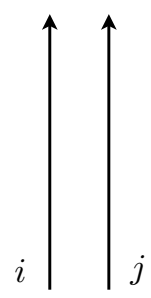

and

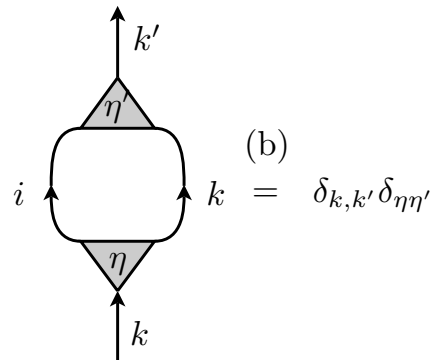

This is unsurprising as they define basis vectors. Indeed, the pictures above are simply the abstraction of the equations

$$
\sum_{\eta, k}|i j ; k, \eta\rangle\langle i j ; k, \eta|=i d \quad \text { and } \quad\left\langle i j ; k, \eta \mid i j, k^{\prime}, \eta^{\prime}\right\rangle=\delta_{k, k^{\prime}} \delta_{\eta, \eta^{\prime}}
$$

satisfied by an orthonormal set of basis vectors.

More generally, a morphism

$$
f: A \rightarrow B
$$

where $A$ and $B$ are understood as compound systems of anyons, is of the form

$$
\bigoplus_{j \in J}\left(\operatorname{Hom}\left(S_{j}, A\right) \stackrel{\operatorname{Hom}\left(1_{S_{j}}, f\right)}{\longrightarrow} \operatorname{Hom}\left(S_{j}, B\right)\right) .
$$


Indeed, lemma 1 tells us that it is not possible to change the global charge from $S_{i}$ to $S_{j}$ for $i \neq j$ as the set of transformation between different simple objects is trivial. We will see some examples of such morphisms in the next section on Fibonacci anyons.

\subsection{Quantum dimension}

In a modular tensor category, the trace of a map $f: A \rightarrow A$ is defined as

$$
\operatorname{Tr}(A): \mathbf{1} \stackrel{i_{A}}{\longrightarrow} A \otimes A^{*} \stackrel{f \otimes 1_{A}}{\longrightarrow} A \otimes A^{*} \stackrel{\delta_{A} \otimes 1_{A}}{\longrightarrow} A^{* *} \otimes A^{*} \stackrel{e_{A^{*}}}{\longrightarrow} \mathbf{1} .
$$

Now, each charge $S_{i}$ has its own dimension. In fact, it is calculated much in the same way than in $\mathbf{F d V e c t} \mathbf{C}_{\mathbb{C}}$. Indeed, it is given by

$$
d_{i}:=\operatorname{Tr}\left(1_{i}\right) .
$$

Pictorially, this is

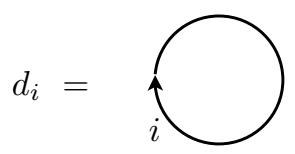

Such a number is called the quantum dimension of $S_{i}$. Before discussing the properties of this number consider the following

Lemma 2. [39] Let $S_{i}, S_{j}$ and $S_{k} \in \mathbf{C}$ be simple objects then

$$
d_{i} d_{j}=N_{k}^{i j} d_{k} .
$$

Proof: We give a pictorial proof:

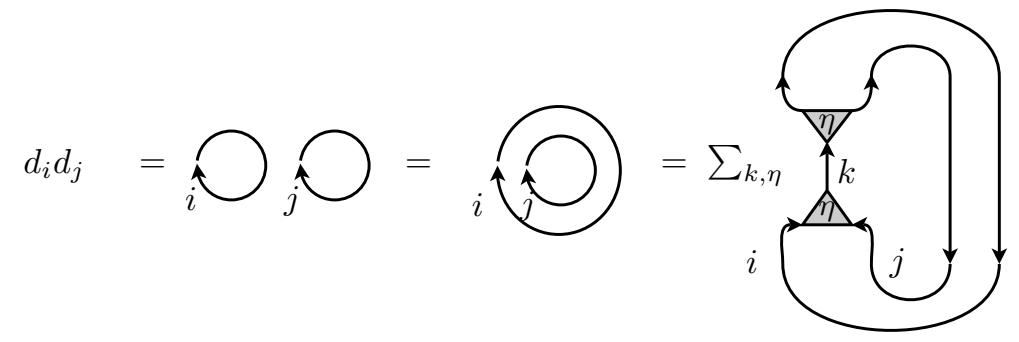

where for the last equality we used (a) from the relations for the morphism underlying the basis vectors. Now, using the topological invariance of the diagram, we get 


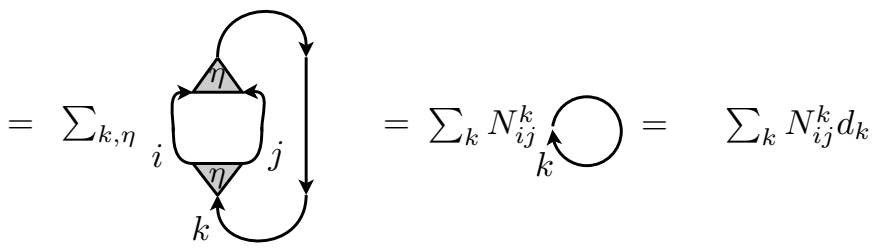

Where for the first equality we used (b) from the same pair of relations.

From this, it is easy to see that the quantum dimension of abelian anyons is always 1 and that, independently of the charge. For a non-abelian anyon of charge $S_{i}$ however, we have $d_{i}>1$.

Example 1. We now give a specific example of quantum dimension via the Fibonacci anyons briefly introduced at the end of section 3. Consider the splitting space

$$
\operatorname{Hom}\left(I, \tau^{\otimes n}\right)
$$

there, $\tau^{\otimes n}$ is the $n$-fold tensor product of $\tau$ 's all bracketed from the left. The dimension of this splitting space can be calculated using the fusion rule $\tau \otimes \tau \simeq \mathbf{1} \oplus \tau$. In doing so, one sees quite quickly that

$$
\tau^{\otimes n} \simeq F_{n-2} \cdot I \oplus F_{n-1} \cdot \tau,
$$

where $F_{m}$ is the $m^{\text {th }}$ Fibonacci number. Now, using lemma 1, we have that

$$
\operatorname{Hom}\left(I, \tau^{\otimes n}\right) \simeq \operatorname{Hom}\left(I, F_{n-2} \cdot I\right) \oplus \operatorname{Hom}\left(I, F_{n-1} \cdot \tau\right) \simeq \operatorname{Hom}\left(I, F_{n-2} \cdot I\right) .
$$

Thus, for the first few values of $n \geq 2$, we have

$$
1,1,2,3,5,8,13,21, \ldots
$$

As this is the sequence of the Fibonacci numbers, the rate of growth must be given by the golden ratio $\phi$.

On the other hand, using our calculations above, we find that $d_{I}=1$ as two charges $I$ fuse trivially. For $d_{\tau}$, we have that

$$
d_{\tau}^{2}=1+d_{\tau}
$$

from which one gets that, again, $d_{\tau}=\phi$, the golden ratio.

The fact that the quantum dimension is an irrational number illustrates that the splitting space obtained via such a set of $\tau$ anyons cannot be decomposed as a tensor product of smaller ones or, in other words, that the information is encoded into global degrees of freedom rather than local ones such as, for instance, the spin of an electron. 


\subsection{The Verlinde formula}

Let $\mathbf{C}$ be a semisimple modular tensor category, we can build the fusion algebra $K$ of $\mathbf{C}$. Without getting into the technical details of such a construction ${ }^{11}$ let us say that it has for basis the set of $x_{j}=\left\langle S_{j}\right\rangle$ for $j \in J$ and for unit $1=x_{0}$. There, $\left\langle S_{j}\right\rangle$ denotes the isomorphism class of $S_{j}$. Of course, the multiplication in $K$ is given by the fusion rules i.e.

$$
x_{i} x_{j}=\sum_{k} N_{i j}^{k} x_{k} .
$$

It turns out that this algebra can be diagonalised i.e. there exists a base in which the multiplication becomes diagonal:

$$
x_{i}^{\prime} x_{j}^{\prime}=\delta_{i j} \alpha x_{j}^{\prime}
$$

where $\alpha$ is a scalar. The matrix that performs this diagonalisation is a renormalised $s$-matrix, the (modular) $S$-matrix which we now define.

Definition 18. Let $d_{j}$ be the quantum dimension of $S_{j} \in|\mathbf{C}|$ and $D$ be the scalar

$$
\sqrt{\sum_{j \in J} d_{j}^{2}}
$$

The S-matrix is

$$
S:=\frac{1}{D} s .
$$

We now have from [5] p. 52 the following

Proposition 3. For a fixed $j \in J$, let $N_{j}$ be the matrix of multiplication by $x_{j}$ in the basis $\left\{x_{j}\right\}$ that is $\left(N_{j}\right)_{a b}=N_{j b}^{a}$ and also, let $D_{j}$ be the diagonal matrix $\left(D_{j}\right)_{a b}=\delta_{a b} S_{i a} / S_{0 a}$, then

$$
S N_{a} S^{-1}=D_{a} .
$$

In fact, this proposition states that the $S$-matrix diagonalises the fusion rules. A more complete discussion along with proofs is given in the source of this proposition and [39].

Now, the previous proposition yields to the well-known result:

\section{Theorem 1. [Verlinde Formula]}

$$
N_{i j}^{k}=\sum_{r} \frac{S_{i j} S_{j r} S_{k^{*} r}}{S_{1 r}} .
$$

In turn, this theorem says that the $S$-matrix is not only related to the braids used to define it but also that given an $S$-matrix, one can calculate the fusion coefficients in $\mathbf{C}$.

$\overline{11}$ This algebra is defined as $K(\mathbf{C}) \otimes_{\mathbb{Z}} \mathbb{K}$ where $K(\mathbf{C})$ is the Grothendieck ring of $\mathbf{C}$. See for instance [5] pp. 32 and 53-54. 


\subsection{Categorical epilogue}

This complete our categorical presentation of the algebra of a family of anyons. Note that even if, in what follows, we use only the semisimple ribbon structure in our description of topological quantum computation, specifying completely the modular tensor category structure was worth the work: indeed, specifying the simple objects, the fusion rules, the pentagon and hexagon axioms, the twist and the $S$-matrix completely determine the topological properties of a species of anyons!

\section{An example: Fibonacci anyons}

The strategy now will be to assume a set of fusion rules of a given species of anyons and solve the various algebraic constraints imposed by the semisimple modular structure. Our intended model to illustrate quantum computation with anyons is the formal semisimple modular tensor category Fib which captures the rules of Fibonacci anyons (see [9, 14, 22, 24, 39] from which the material of this section is derived).

- Fibonacci anyons have only two charges: $\mathbf{1}$ and $\tau$, where $\mathbf{1}$ is the trivial charge,

- Both are their own anti-charge,

- They satisfies the following fusion rules:

$$
\begin{aligned}
& \mathbf{1} \otimes \mathbf{1} \simeq \mathbf{1} \\
& \mathbf{1} \otimes \tau \simeq \tau \otimes \mathbf{1} \simeq \tau \\
& \tau \otimes \tau \simeq \mathbf{1} \oplus \tau
\end{aligned}
$$

Categorically, this says that the semisimple modular tensor category Fib has

- Two simple objects $\mathbf{1}$ and $\tau$ where $\mathbf{1}$ is the tensor unit,

- That they are their own dual i.e.: $\mathbf{1}^{*}=\mathbf{1}$ and $\tau^{*}=\tau$ and,

- That $\mathbf{1}$ and $\tau$ satisfy the fusion rules given above.

Let us inspect the fusion rules. While the two first trivially hold, the third one says that the charge resulting from the fusion of two anyons of charge $\tau$ is either $\mathbf{1}$ or $\tau$. It is precisely this third rule that tells us that our anyons are non-abelian as they can fuse in two distinct ways.

Now, back to our model, consider three anyons of charge $\tau$ all lined up $(\tau \otimes \tau) \otimes \tau$ and let them fuse in the order fixed by the bracketing. Such a process is algebraically described by:

$$
\begin{aligned}
(\tau \otimes \tau) \otimes \tau & \simeq(\mathbf{1} \oplus \tau) \otimes \tau \\
& \simeq(\mathbf{1} \otimes \tau) \oplus(\tau \otimes \tau) \\
& \simeq \tau \oplus(\mathbf{1} \oplus \tau) \\
& \simeq \mathbf{1} \oplus 2 \cdot \tau .
\end{aligned}
$$


Hence, the fusion process for three $\tau$ anyons yields a final charge $\tau$ in 2 different ways or $\mathbf{1}$ in a single way. These three scenarios depict as

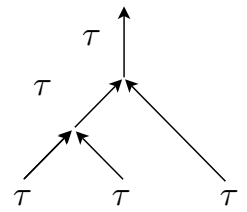

or

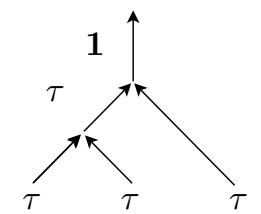

or

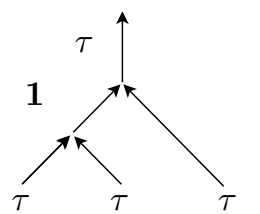

We now pass to the context of finite-dimensional complex vector spaces via the splitting spaces whose basis vectors are dual to the fusion states described above. Consider

$$
\begin{aligned}
\operatorname{Hom}(b,(\tau \otimes \tau) \otimes \tau) & \simeq \operatorname{Hom}(b, \mathbf{1} \oplus 2 \cdot \tau) \\
& \simeq \operatorname{Hom}(b, \mathbf{1}) \oplus \operatorname{Hom}(b, 2 \cdot \tau) \text { and as } 2 \cdot \tau:=\tau \oplus \tau \text { this is } \\
& \simeq \operatorname{Hom}(b, \mathbf{1}) \oplus 2 \cdot \operatorname{Hom}(b, \tau) .
\end{aligned}
$$

Now, using lemma 1 in conjunction with the property that for any $b \in\{\mathbf{1}, \tau\}$, $\operatorname{End}(b) \simeq \mathbb{C}$; if we set $b=\mathbf{1}$, then (5) is isomorphic to $\mathbb{C} \oplus 2 \cdot 0$. Conversely if $b=\tau$, then it is isomorphic to $0 \oplus 2 \cdot \mathbb{C}$.

From this, we conclude that considering the space of states with global charge $b \in\{\mathbf{1}, \tau\}$ is the same as considering

$$
\operatorname{Hom}(b,(\tau \otimes \tau) \otimes \tau)
$$

In its turn, such a consideration fixes either of the splitting spaces $\mathbb{C}$ or $2 \cdot \mathbb{C}:=$ $\mathbb{C}^{2}$ as orthogonal subspaces of $\mathbb{C}^{3}$, the topological space representing our triple of anyons. It is within this two-dimensional complex vector space that we will simulate our qubit. Indeed, if $b=\tau$, we are left with two degrees of freedom which are the two possible outputs of the second splitting.

Remark 8. It is worth stressing that it takes three anyons of charge $\tau$ to simulate a single qubit. Moreover, we shall see later that braiding these anyons together simulates a unitary transformation on such a simulated qubit.

Remark 9. Since Fib is rigid, we can apply proposition 1. We have

$$
\begin{aligned}
\operatorname{Hom}(\tau,(\tau \otimes \tau) \otimes \tau) & \simeq \operatorname{Hom}(\mathbf{1} \otimes \tau,(\tau \otimes \tau) \otimes \tau) \\
& \simeq \operatorname{Hom}(\mathbf{1},((\tau \otimes \tau) \otimes \tau) \otimes \tau) .
\end{aligned}
$$

Comparing this fact with what we got in example 1, we see that these two encodings are essentially the same. It is because of this that some authors, for instance J. Preskill in [39], prefer to encode their qubits within a quadruple of anyons of individual charge $\tau$ with global charge $\mathbf{1}$ instead. We choose the former to align with the work of Bonesteel et al. [9] that we will explain in section 6 . 
Now that we have an expression for the topological spaces in Fib, it will be handy to fix a basis for them. Using the diagram given in the section on splitting spaces, we get

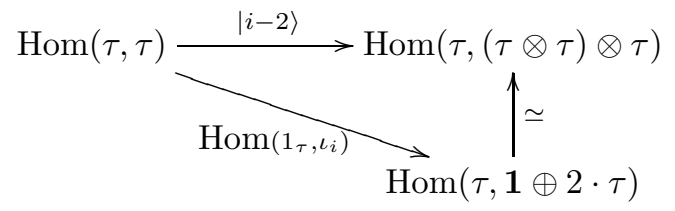

with $i \in\{2,3\}$ and where the vertical isomorphism is built from the fusion rules.

Analogously, the basis vector $|N C\rangle$ spanning the one dimensional fusion space is defined as

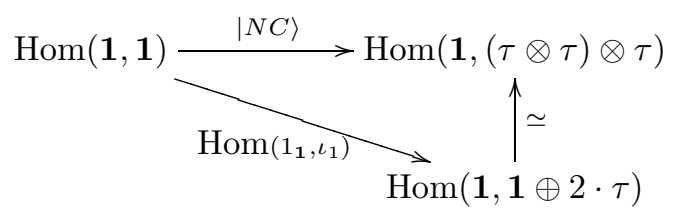

It is labeled $N C$ for Non-Computational. Indeed, the superposition of $|N C\rangle$ with $|0\rangle$ or $|1\rangle$ is prohibited.

\subsection{The F-matrix}

In order to ensure consistency of the model Fib, splitting has to be associative as expressed categorically via the pentagon axiom from the monoidal structure.

There are two splitting spaces that can be obtained from a triple of anyons i.e.: $(\tau \otimes \tau) \otimes \tau$ and $\tau \otimes(\tau \otimes \tau)$. The basis vectors for these two splitting spaces are - of course - related by a unitary transformation called the $F$-matrix acting on the splitting spaces and defined via the natural transformation $\alpha$ in the following way:

$$
F: \operatorname{Hom}(W,(S \otimes T) \otimes U) \stackrel{\operatorname{Hom}\left(1_{W}, \alpha_{S, T ; U}\right)}{\longrightarrow} \operatorname{Hom}(W, S \otimes(T \otimes U)) .
$$

There, $S, T, U$ and $W \in\{\mathbf{1}, \tau\}$.

Using splitting diagrams, we have:

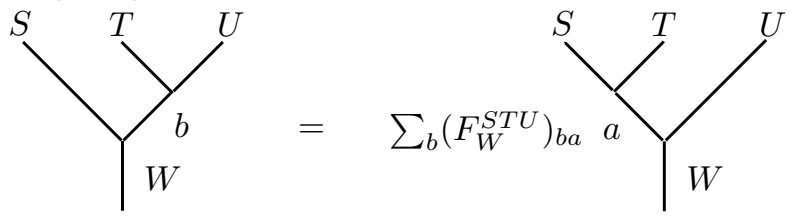


Considering the splitting diagram for fixed $a$ and $W$ as a basis vector, this is nothing but the matrix expression of $F$. In order to obtain a solution for the $F$-matrix, we need to recast the pentagon axiom from the monoidal structure in this context in such a way that we obtain a matrix equation. Consider

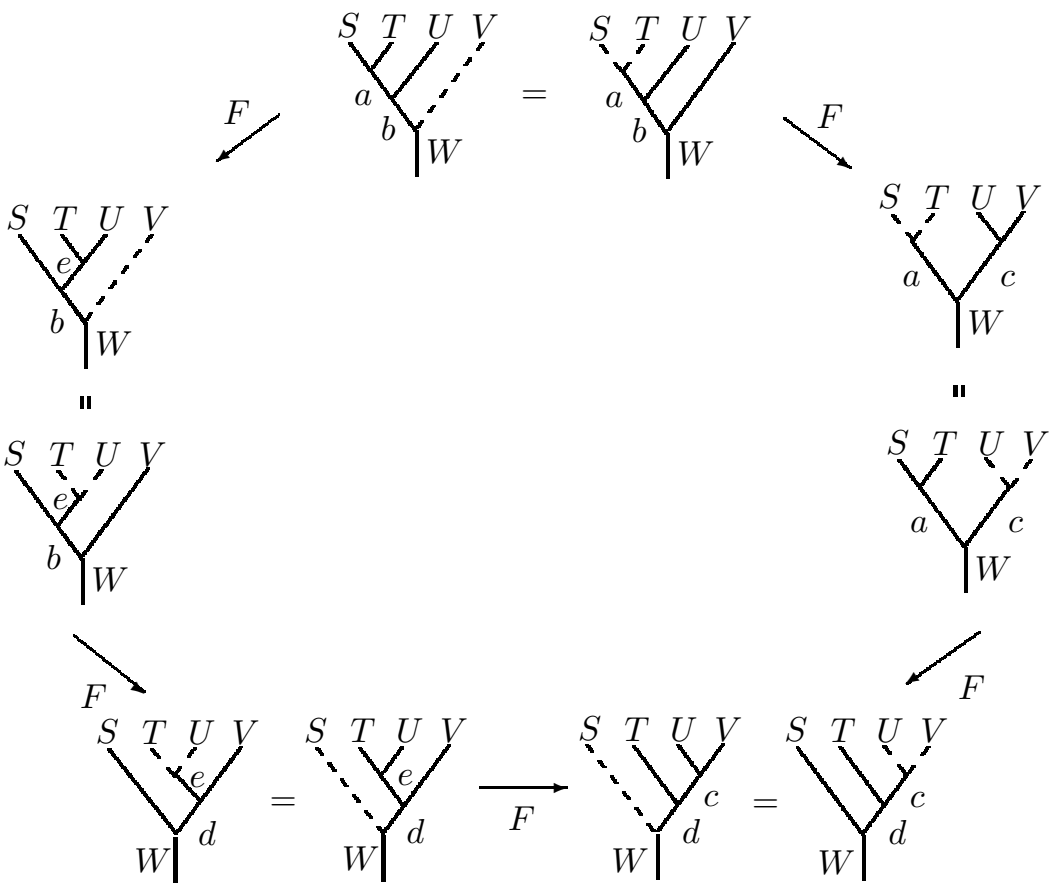

There, we explicitly expressed where $F$ was acting on the splitting states via subdiagrams drawn in solid lines. Passing to the underlying pentagon axiom is made via the expression of $F$ given in equation 6 .

Now, equating both sides of the diagram yields

$$
\left(F_{W}^{S T c}\right)_{d a}\left(F_{W}^{a U V}\right)_{c b}=\sum_{e}\left(F_{d}^{T U V}\right)_{c e}\left(F_{W}^{S e V}\right)_{d b}\left(F_{b}^{S T U}\right)_{e a}
$$

Solving this in conjunction with a given set of fusion rules yields the $F$-matrix. To solve such an equation, one has to fix the labels for all the possible states in the splitting basis and solve the resulting system of equations.

In Fib, for a triple of anyons of charge $\tau$, the trivial charge can split into such a triple in only one way. In this particular case, the $F$-matrix is

$$
F_{1}^{\tau \tau \tau}=[1]
$$

as the first splitting must yields $\tau \otimes \tau$.

Conversely, if the initial charge is $\tau$ then, the splitting process can occur in two distinct manners. In order to get the $F$-matrix, we must use equation 7 . 
For instance, a possible splitting scenario occurs when one fixes $a=\mathbf{1}=c$ and $d=\tau=b$. Using this with 7 gives:

$$
\begin{aligned}
\left(F_{\mathbf{1}}^{\tau \tau \mathbf{1}}\right)_{\tau \mathbf{1}}\left(F_{\mathbf{1}}^{\mathbf{1} \tau \tau}\right)_{\mathbf{1} \tau} & =\sum_{e \in\{\mathbf{1}, \tau\}}\left(F_{\tau}^{\tau \tau \tau}\right)_{\mathbf{1} e}\left(F_{\mathbf{1}}^{\tau e \tau}\right)_{\tau \tau}\left(F_{\tau}^{\tau \tau \tau}\right)_{e \mathbf{1}} \\
1 & =F_{\mathbf{1 1}}^{2}+F_{\tau \mathbf{1}} F_{\mathbf{1} \tau} .
\end{aligned}
$$

Using this, the other consistency relations and the fact that $F$ is unitary, we find:

$$
F_{\tau}^{\tau \tau \tau}=\left[\begin{array}{ll}
F_{11} & F_{1 \tau} \\
F_{\tau \mathbf{1}} & F_{\tau \tau}
\end{array}\right]=\left[\begin{array}{cc}
\phi^{-1} & \sqrt{\phi^{-1}} \\
\sqrt{\phi^{-1}} & -\phi^{-1}
\end{array}\right]
$$

where $\phi$ is the golden ratio.

Finally, combining the results for $F_{\tau}^{\tau \tau \tau}$ and $F_{1}^{\tau \tau \tau}$ yields

$$
F=\left[\begin{array}{c|cc}
1 & 0 & 0 \\
\hline 0 & \phi^{-1} & \sqrt{\phi^{-1}} \\
0 & \sqrt{\phi^{-1}} & -\phi^{-1}
\end{array}\right]
$$

which is also unitary. The lower-right block induces a change of basis on the 2-dimensional splitting space while the upper-left block is the trivial transformation on the one-dimensional splitting space.

\subsection{Braiding anyons}

We now express what will be the consequence of exchanging two anyons on the splitting space. As such an exchange is represented categorically by a braiding, this will yield a representation of the braid group in the splitting space.

\section{The R-matrix}

The game here is very similar to the one for the $F$-matrix except that we use the hexagon axiom from the braided monoidal structure instead. The $R$-matrix is a morphism

$$
R: \operatorname{Hom}(W,(S \otimes T) \otimes U) \stackrel{\operatorname{Hom}\left(1_{W}, \sigma_{S, T} \otimes 1_{U}\right)}{\longrightarrow} \operatorname{Hom}(W,(T \otimes S) \otimes U) .
$$

or, using splitting diagrams:

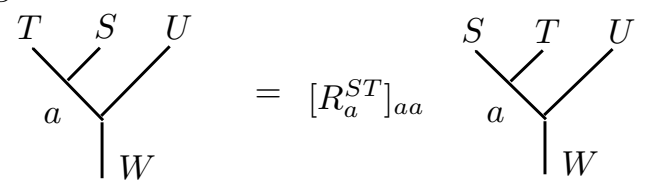


We already have the $F$-matrix thus, the hexagon needs to be solved only for the $R$-matrix. Recasted with splitting diagrams, the hexagon axiom becomes:
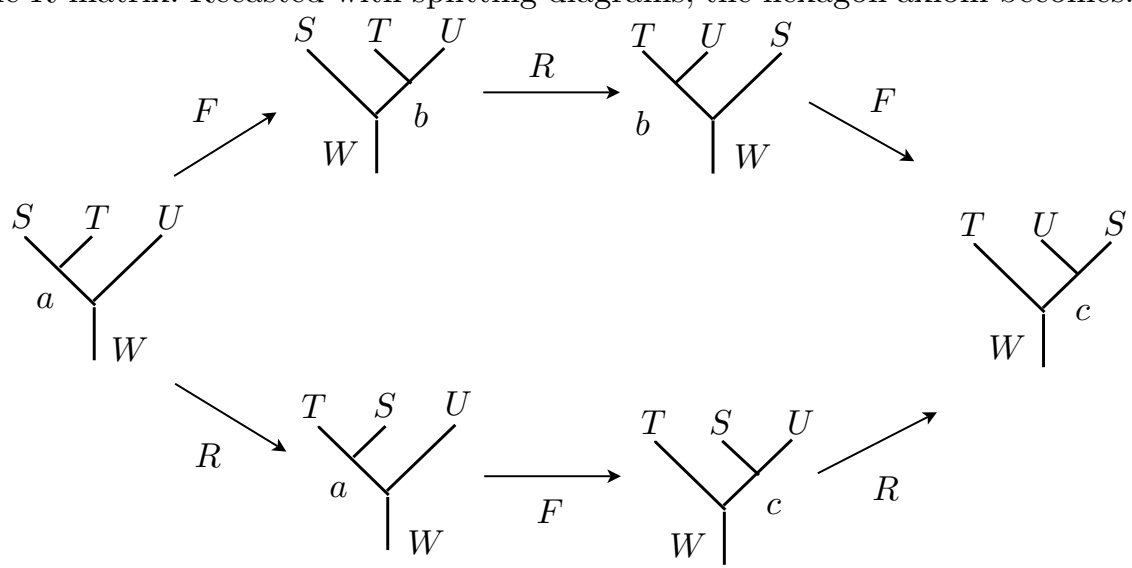

Writing it as a matrix equation yields

$$
R_{c}^{S U}\left(F_{W}^{T S U}\right)_{c a} R_{a}^{S T}=\sum_{b}\left(F_{W}^{T U S}\right)_{b c} R_{W}^{S b}\left(F_{W}^{S T U}\right)_{b a} .
$$

For a triple of anyons with charge $\tau$, explicit calculations of the R-matrix yields:

$\left[\begin{array}{c|cc}-e^{-2 i \pi / 5} & 0 & 0 \\ \hline 0 & e^{-4 i \pi / 5} & 0 \\ 0 & 0 & -e^{-2 i \pi / 5}\end{array}\right]$

Such a diagonal form is not surprising: whether the global charge of a couple is 1 (resp. $\tau$ ), it must remain so even if we exchange the two components of the pair.

\section{The B-matrix}

The $R$-matrix provided in the previous section give us a way to exchange the two leftmost anyons in a set of three. We now need a way to find the matrix that exchanges the two rightmost anyons, this will be the $B$-matrix and is defined as

$$
\begin{aligned}
& \operatorname{Hom}(W,(S \otimes T) \otimes U) \longrightarrow \operatorname{Hom}(W,(S \otimes U) \otimes T) \\
& \operatorname{Hom}\left(1_{W}, \alpha_{S, T ; U}\right) \downarrow \quad \uparrow \operatorname{Hom}\left(1_{W}, \alpha_{S, U ; T}^{-1}\right) \\
& \operatorname{Hom}(W, S \otimes(T \otimes U)) \underset{\operatorname{Hom}\left(1_{W}, 1_{S} \otimes \sigma_{T, U}\right)}{\longrightarrow} \operatorname{Hom}(W, S \otimes(U \otimes T))
\end{aligned}
$$

As we found both the $F$ and the $R$ matrix in $\mathbf{F i b}$, we can compute the $B$ matrix as

$$
B:=F^{-1} R F
$$




\section{Universal quantum computation with Fibonacci anyons}

The basic idea to simulate quantum computation with anyons is given by the following steps:

1. Consider a compound system of anyons. We initialise a state in the splitting space by fixing the charges of subsets of anyons according to the way they will fuse. This determines the basis state in which the computation starts.

2. We braid the anyons together, it will induce a unitary action on the chosen splitting space.

3. Finally, we let the anyons fuse together and the way they fuse determines which state is measured and this constitutes the output of our computation.

\section{Simulating qubits}

First, the topological space for such a triple is a pair $\left\langle\mathbb{C}, \mathbb{C}^{2}\right\rangle$ where the 2 -dimensional space is spanned by the fusion states

$$
|0\rangle:=|(\tau \otimes \tau) \otimes \tau ; \tau, 1\rangle \quad \text { and } \quad|1\rangle:=|(\tau \otimes \tau) \otimes \tau ; \tau, 2\rangle,
$$

and the space of dimension one is spanned by:

$$
|N C\rangle:=|(\tau \otimes \tau) \otimes \tau ; \mathbf{1}, 1\rangle .
$$

Of course, the simulation of a qubit will occur on the 2-dimensional space spanned by $\{|0\rangle,|1\rangle\}$. Compound system of two or more qubits will be given by the compound system of such a triple of anyons. Note that even if we fix the global charge of the triple as $\tau$, in the real world, it is possible that we may still measure 1. These errors are known as "leakage errors" as there is some unexpected "leaks" into another splitting space.

\section{Quantum computation}

To perform actual quantum computation, it seems at first glance that we have two problems:

1. First, we would like to apply any gate on our simulated qubits but we have only the two braiding matrices and their inverses.

2. Second, even if we solve our first problem, it remains that this is not enough to quantum compute. Indeed, we also need a two-qubit gate.

We answer these. First, a composition of length $l$ of $R$ - and $B$-matrices and their inverses can get arbitrarily close to any element of $S U(2)$ and that, with $l$ reasonably small. This is a consequence of the fact that our matrices together with their inverse satisfies the Solovay-Kitaev theorem, see [36] pp. 617-624 for a precise statement and a proof. 
We now address the second problem. Following the works of Bonesteel et al. in [9], we explain how to build a CNOT gate for our anyons; for this we will need two triplets of $\tau$ anyons; one of them will act as our test qubit while the other will be the target qubit. The idea is relatively simple: we need to intertwine a pair of quasi-particles from the first triplet - the control pair with the target triplet without disturbing it; as the braid operators are dense in $S U(2)$, we will arrange such an intertwining so that its representation in $S U(2)$ is close enough to to the identity. The next thing is to implement a NOT - actually a $i \cdot \mathbf{N O T}$ - by braiding our two anyons of the control pair with those of the target triple. Finally, we extract the control pair from the second triplet - again - without disturbing it. Now, the key point is the following: a braiding involving the trivial charge $\mathbf{1}$ with an anyon of arbitrary charge does not change anything. Thus, when measuring the control pair, the $i \cdot$ NOT will occur if and only if the two anyons from the control pair fuse as an anyon of charge $\tau$; otherwise the control pair only induces a trivial change on the system.

a) Consider the following braiding:

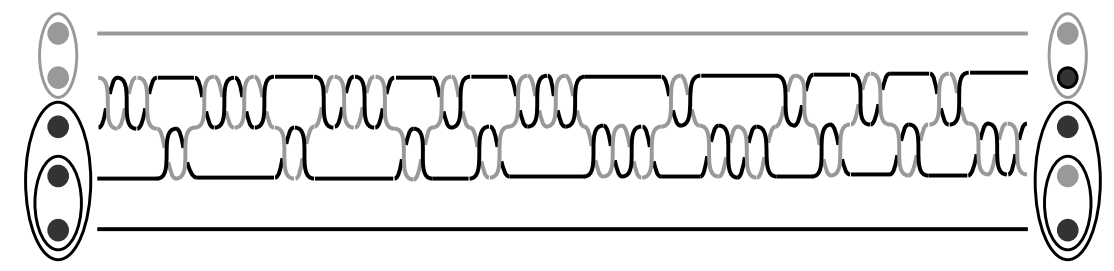

As an action on the splitting space of the three anyons involved, this is, in the same order as depicted in the picture:

$$
B^{3} R^{-2} B^{-4} R^{2} B^{4} R^{2} B^{-2} R^{-2} B^{-4} R^{-4} B^{-2} R^{4} B^{2} R^{-2} B^{2} R^{2} B^{-2} R^{3} \sim\left(\begin{array}{cc|c}
1 & 0 & 0 \\
0 & 1 & 0 \\
\hline 0 & 0 & 1
\end{array}\right)
$$

This tells us how the given braid insert an anyon within a triplet without disturbing it. In fact, this stresses the distinction between the dynamics of the anyons and the consequences on the splitting space. Indeed, even if we disturbed the initial configuration of anyons via multiple braidings, the effect on the splitting space is approximately the identity.

b) Now, we implement an $i \cdot$ NOT as the following braid:

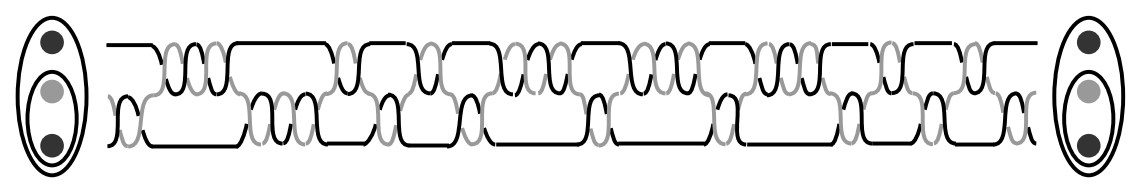

The unitary acting on the splitting space of the initial triple is given by: 


$$
R^{-2} B^{-4} R^{4} B^{-2} R^{2} B^{2} R^{-2} B^{4} R^{-2} B^{4} R^{2} B^{-4} R^{2} B^{-2} R^{2} B^{-2} R^{-2} \sim\left(\begin{array}{cc|c}
0 & i & 0 \\
i & 0 & 0 \\
\hline 0 & 0 & 1
\end{array}\right)
$$

This combination of braids tells us how to implements a $i \cdot$ NOT gate on the two dimensional fusion space of our triple of anyons. Again, this gate is approximated.

c) Finally, the $i \cdot$ CNOT gate acting on two topological qubits is realised as follows:

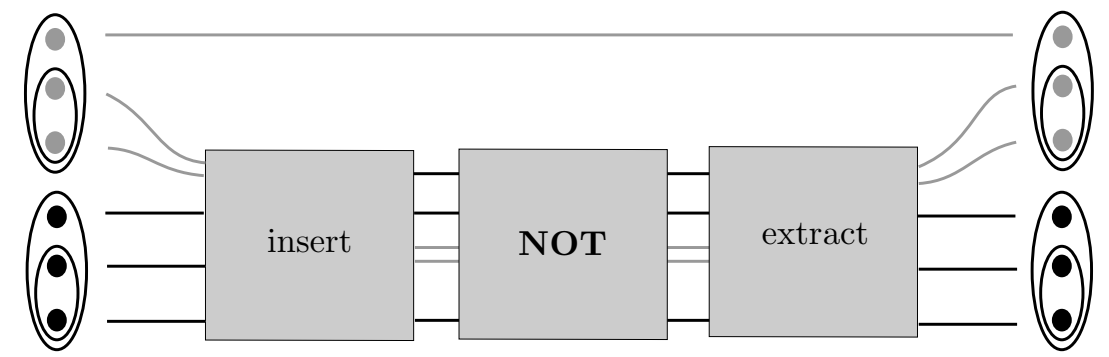

First, instead of inserting 1 anyon, we insert a couple that will be used as a test couple and that in the very same manner as described in a) - as these two will fuse together yielding either $\mathbf{1}$ or $\tau$, this is exactly what we want. Secondly, we apply the $i$.NOT-gate computed in b). Finally, we extract the control pair returning it to its original position by applying the insertion procedure in reverse order. This is done, again, without disturbing the triple at stance here.

We claim that this implements a CNOT. Indeed, the test couple can fuse in two ways. If it fuse as $\mathbf{1}$, then nothing happens as $\mathbf{1}$ is the trivial charge. If it fuse as $\tau$, then we effectively apply the $i$. NOT gate computed in b).

Interestingly, we may replace the $i$.NOT by any other braid thus obtaining a way to perform other controlled operations give such a pair of topological qubits. It turns out that this gate together with the $R$ and $B$ matrices form a universal set of quantum gates [10] as $i \cdot$ CNOT is entangling.

\section{Conclusions}

As we noted in the introduction of section 4 the results that we presented here are not new. We gave an explicit description of the algebra of anyons in terms of modular categories, a description that is given in mathematical physics papers that may not be accessible to everyone.

The very rich nature of this subject makes the task of writing a complete introduction concisely almost impossible therefore, to complement our attempt, we now give some additional pointers to the litterature. On the physical side, Frank Wilczek edited Fractional statistics and anyon superconductivity [52], 
a book which comprises papers explaining central concepts related to anyons. This list wouldn't be complete without A. Kitaev's paper Anyons in an exactly solved model and beyond [29] where the reader can find another introduction on the algebra of anyons with a more physical flavour in appendix E.

On the quantum computing side, there is a growing interest in topological quantum computation due to the fact that such a model provides a more robust form of quantum computation. Some papers which emphasise topological quantum computation are [3] and [42]. Perhaps closer to the essence of the present paper are the works of Freedman, Kitaev, Larsen and Wang presented in $[21]$ and $[22]$.

Of course, apart from modular tensor categories, there are other links to draw between topology and quantum mechanics or quantum computing; for instance, the works of Louis H. Kaufman and Samuel J. Lomonaco Jr., in particular [26] in which they describe the role of braiding in quantum computing. There is also the work of S. Abramsky [1] that describes connections between knot theory, categorical quantum mechanics, logic and computation.

Finally, although the aim of this paper was to draw parallels between categorical structures and anyons, there is still work to do; for instance, our exposition was oriented towards FdHilb, the category of finite-dimensional Hilbert spaces. However, $\dagger$-compact categories [2] constitute a correct framework to describe quantum mechanics and it would be interesting to see the benefits of describing topological quantum computation within this more abstract context.

\section{Acknowledgments}

The authors would like to thank Samson Abramsky, Michel Boyer, Ross Duncan, Rafael Sorkin, Colin Stephen, Benoit Valiron and Jamie Vicary for useful discussions.

\section{References}

1. S. Abramsky. Temperley-lieb agebra: from knot theory to logic and quantum computation via quantum mechanics. In G. Chen, L. Kauffman, and S. Lomonaco, editors, Mathematics of quantum computing and technology, pages 515-558. Taylor and Francis, 2007.

2. S. Abramsky and B. Coecke. A categorical semantics of quantum protocols. Proceedings of the 19th Annual IEEE Symposium on Logic in Computer Science (LiCS`04), IEEE Computer Science Press., 2004.

3. D. Aharonov, V. Jones, and Z. Landau. A polynomial quantum algorithm for approximating the jones polynomial. In STOC '06: Proceedings of the thirtyeighth annual ACM symposium on Theory of computing, pages 427-436, New York, NY, USA, 2006. ACM. 
4. B. Bakalov and A. Kirilov Jr. On the Lego-Teichmüller game. Transf. Groups, (5):7-44, 2000.

5. B. Bakalov and A. Kirillov. Lectures on tensor categories and modular functors. American Mathematical Society in University Lecture Series, 2001.

6. A. P. Balachandran, A. Daughton, Z. Gu, R. D. Sorkin, G. Marmo, and A. M. Srivastava. Spin-statistics theorems without relativity or field-theory. Int. J. Mod. Phys., A 8:2993-3044, 1993.

7. A. P. Balachandran, W. D. McGlinn, L. O'Raifeartaigh, S.Sen, R.D.Sorkin, and A.M.Srivastava. Topological spin-statistics theorem for strings. Mod. Phys. Lett., A7:1427-1442, 1992.

8. M. V. Berry and J. M. Robbins. Indistinguishability for quantum particles: spin, statistics and the geometric phase. Proc. Roy. Soc. A, 453(1963):17711790, August 1997.

9. N. E. Bonesteel, L. Hormozi, and G. Zikos. Braid topologies for quantum computation. Phys. Rev. Lett., 95(140503), 2005.

10. M. J. Bremner, C. M. Dawson, J. L. Dodd, A. Gilchrist, A. W. Harrow, D. Mortimer, M. A. Nielsen, and T. J. Osborne. Practical scheme for quantum computation with any two-qubit entangling gate. 89(247902), 2002.

11. N. Burgoyne. On the connection between spin and statistics. Nuovo Cimento, VIII(4):607-609, 1958.

12. B. Coecke and É. O. Paquette. Categories for the practising physicist. In B. Coecke, editor, ?, page ? Springer-Verlag, ?

13. L. Crane. 2-d physics and 3-d topology. Comm. Math. Phys., (135):615-640, 1991.

14. S. Das Sarma, M. Freedman, C. Nayak, S. H. Simon, and A. Stern. Non-abelian anyons and topological quantum computation. ArXiv e-prints, 707, July 2007.

15. J. S. deWet. On the connection between the spin and statistics of elementary particles. Phys. Rev., 57:646-652, 1940. Ph.D. thesis, Princeton University 1939.

16. H. F. Dowker and R. D. Sorkin. A spin-statistics theorem for certain topological geons. Class. Quantum Grav., 15(5):1153-1167, 1998.

17. I. Duck and E. C. G. Sudarshan. Towards an understanding of the spin-statistics theorem. Am. J. Phys., 66(4):284-303, 1998.

18. I. Duck, E. C. G. Sudarshan, and Arthur S. Wightman. Pauli and the spinstatistics theorem. American Journal of Physics, 67(8):742-746, 1999. Review by Wightman of the book by Duck and Sudarshan.

19. M. Fierz. Uber die relativische Theorie kräftfreir Teilchen mit beliebigem spin. Helv. Phys. Acta, 12(3), 1939.

20. D. Finkelstein and J. Rubinstein. Connection between spin statistics and kinks. J. Math. Phys., 9(11):1762-1779, 1968.

21. M. H. Freedman, A. Kitaev, and Z. Wang. Simulation of topological field theories by quantum computers. Comm. Math. Phys., (227):587-603, 2002.

22. M. H. Freedman, M. Larsen, and Z. Wang. A modular functor which is universal for quantum computation. Comm. Math. Phys., (227):605-622, 2002.

23. P. Freyd. Abelian categories. Harper and Row, 1964.

24. L. Hormozi, G. Zikos, and N. E. Bonesteel. Topological quantum compiling. Physical Review B, 75:165310, 2007.

25. A. Joyal and R. Street. Braided tensor categories. Advances in Math., (102):20 78, 1993.

26. L. H. Kauffman and S. J. Lomonaco Jr. Braiding operators are universal quantum gates. New J. of Phys., 6(134), 2004. 
27. T. Khono. Topological invariants for 3-manifolds using representations of the mapping class groups i. Topology, (31):203-230, 1992.

28. A. Kitaev. Fault-tolerant quantum computation by anyons. Ann. Phys., 303(1):3-20, 2003.

29. A. Kitaev. Anyons in an exactly solved model and beyond. 2006.

30. K. v. Klitzing, G. Dorda, and M. Pepper. New method for high-accuracy determination of the fine-structure constant based on quantized hall resistance. Phys. Rev. Lett., 45(6):494-497, Aug 1980.

31. S. Mac Lane. Categories for the working mathematician. Springer graduate text in mathemathics, Vol. 5. Springer-Verlag, New-York, 1998.

32. R. B. Laughlin. Quantized hall conductivity in two dimensions. Phys. Rev. B, 23(10):5632-5633, May 1981.

33. J. M. Leinaas and J. Myrheim. On the theory of identical particles. Il Nuovo Cimento, 37B:1-23, 1977.

34. G. Lüders and B. Zumino. Connection between spin and statistics. Phys. Rev., 110:1450-1453, 1958.

35. A. Messiah. Quantum Mechanics (Vol. 1). Jonhn Wiley \& Sons, 1966.

36. M. A. Nielsen and I. L. Chuang. Quantum computation and quantum information. Cambrige University Press, 2000.

37. W. Pauli. The connection between spin and statistics. Phys. Rev., 58:716-722, 1940.

38. W. Pauli. On the connection between spin and statistics. Prog. Theor. Phys., 5(4):526-543, 1950.

39. J. Preskill. Lectures notes physics 219 / computer science 219 quantum computation. 2004.

40. N. Yu. Reshetikhin and V. G. Tuarev. Ribbon graphs and their invariants derived from quantum groups. Comm. Math. Phys., 127:1-26, 1990.

41. N. Yu. Reshetikhin and V. G. Tuarev. Invariants of 3-manifolds via link polynomials and quantum groups. Invent. Math., 103:547-597, 1991.

42. S. Das Sarma, M. Freedman, and C. Nayak. Topologically-protected qubits from a possible non-abelian fractional quantum hall state. Physical Review Letters, 94:166802, 2005.

43. J. Schwinger. Spin, statistics and the TCP theorem and Addendum. Proc. Natl. Acad. Sci. USA, 44:223-228,617-619, 1958.

44. R. D. Sorkin. Particle-statistics in three dimensions. Phys. Rev. D, 27:17871797, 1983.

45. H. Störmer. Nobel Lecture: Physics 1996-2000, chapter The fractional quantum Hall effect, pages 295-326. World Scientific, 2002.

46. R. F. Streater and A. S. Wightman. PCT, Spin, Statistics and All That. Benjamin, New York, 1964.

47. V. G. Tuarev. Quantum invariant of knots and 3-manifolds. W. de Gruyter, Berlin, 1994.

48. A. S. Wightman. Quantum field theory in terms of vacuum expectation values. Phys. Rev., 101(2):860-866, January 1956.

49. A. S. Wightman. The spin-statistics connection: some pedagogical remarks in response to neuenschwander's question. Electronic Journal of Differentail Equations, pages 207-213, 2000. Conference on Mathematical Physics and Quantum Field Theory.

50. F. Wilczek. Magnetic flux, angular momentum, and statistics. Phys. Rev. Lett., 48(17):1144-1146, April 1982. 
51. F. Wilczek. Quantum mechanics of fractional-spin particles. Phys. Rev. Lett., 49(14):957-959, Oct 1982.

52. F. Wilczek, editor. Fractional statistics and anyon superconductivity. World Scientific, New Jersey, 1990. 